\title{
RECENT DEVELOPMENTS IN INVERSE ACOUSTIC SCATTERING THEORY
}

\author{
DAVID COLTON*, JOE COYLE ${ }^{\dagger}$, AND PETER MONK
}

\begin{abstract}
We survey some of the highlights of inverse scattering theory as it has developed over the past fifteen years, with emphasis on uniqueness theorems and reconstruction algorithms for time harmonic acoustic waves. Included in our presentation are numerical experiments using real data and numerical examples of the use of inverse scattering methods to detect buried objects.
\end{abstract}

Key words. Inverse problems, scattering theory.

AMS subject classifications. 35P25, 35R30, 76Q05

1. Introduction. The field of inverse problems is a relatively new area of mathematical research, having its origins in the fundamental papers of Tikhonov in the mid-1960's. As with any new area of mathematics, one can ask the question, why did it start when it did and not sooner? In the case of inverse problems, the answer is one of historical prejudice meeting scientific pressure. The historical prejudice dates back to Hadamard, who claimed that the only problems of physical interest were those that had a unique solution depending continuously on the given data. Such problems were called well-posed, and problems that were not well-posed were labeled ill-posed [30]. In particular, ill-posed problems connected with partial differential equations of mathematical physics were considered to be of purely academic interest and not worthy of serious study. In the meantime, the success of radar and sonar during the Second World War caused scientists to ask if more could be determined about a scattering object than simply its location. Such problems are in the category of inverse scattering problems, and it was slowly realized that these problems, although of obvious physical interest, were ill-posed. However, due to the lack of a mathematical theory of inverse problems, together with limited computational capabilities, further progress was not possible.

This situation was dramatically changed in the mid-1960's with the introduction of regularization methods for linear ill-posed problems by Tikhonov (see [74]). In particular, Tikhonov considered ill-posed operator equations of the form

$$
A \varphi=f,
$$

where $A: X \rightarrow Y$ is a compact operator mapping a Banach space $X$ into a Banach space $Y$, and he noted that since $A$ is compact, this equation is ill-posed. A regularized solution of (1.1) is then found by minimizing the Tikhonov functional

$$
\|A \varphi-f\|^{2}+\gamma\|\varphi\|^{2}
$$

where $\gamma>0$ is called the regularization parameter. It can be shown [38], [45] that for each $\gamma>0$ there exists a unique minimizer $\varphi_{\gamma}$ to (1.2) and that $\varphi_{\gamma}$ depends continuously on $f$. The choice of regularization parameter is obviously crucial if $\varphi_{\gamma}$

\footnotetext{
*Department of Mathematical Sciences, University of Delaware, Newark DE 19716,USA. colton@math.udel.edu

${ }^{\dagger}$ Department of Mathematics, University of Strathclyde, Glasgow, Scotland

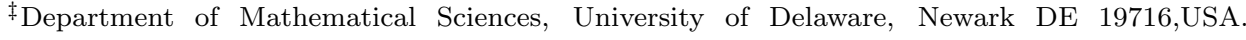
monk@math.udel.edu
} 
is to approximate a solution to (1.1), and a method for choosing this parameter as a function of the noise level in $f$ was given by Morozov (cf. [38], [45]). Assuming $A$ is known exactly and $f$ is noisy, $\gamma$ is chosen such that the defect $\|A \varphi-f\|$ is the same order of magnitude as the noise level, leading to a nonlinear equation for the determination of the parameter $\gamma$ where the equation depends on the noise level. For the case when the operator $A$ is noisy, similar considerations apply (see Section 2 ). The further development of the mathematical theory of linear ill-posed problems by Tikhonov and his school in Russia and by Keith Miller and others in the United States, together with the rapid development of computing facilities, set the stage for the subsequent mathematical investigation of the inverse scattering problem.

1.1. The Inverse Scattering Problem. Before proceeding further we need to explain in more detail what we mean by the inverse scattering problem. In this paper, for purposes of exposition, we will primarily restrict our attention to the case of acoustic waves. In particular, consider an acoustic wave propagating in a homogeneous isotropic medium. In the absence of any inhomogeneities, the wave will continue to propagate and nothing of physical interest will happen. However, if there are inhomogeneities present, then the wave will be "scattered" and we can express the total field as the sum of the original "incident" wave and the "scattered" wave. The behavior of the scattered wave will depend on both the incident wave and the nature of the inhomogeneities in the medium. The direct problem is, given this information, to find the scattered wave and in particular its behavior at large distances from the inhomogeneities, i.e. its "far field" behavior. The inverse problem takes this answer to the direct scattering problem as its starting point and asks what is the nature of the inhomogeneities which gave rise to such a far field behavior.

To be more precise, consider the scattering of a time harmonic acoustic wave by a bounded object in three dimensional Euclidean space $\mathbb{R}^{3}$, and assume the object $D$ is situated in a homogeneous isotropic medium with density $\rho$ and speed of sound $c$. The wave motion can be determined from a velocity potential $U=U(x, t), x \in \mathbb{R}^{3} \backslash \bar{D}$, which in the linearized theory satisfies the wave equation

$$
\frac{\partial^{2} U}{\partial t^{2}}=c^{2} \Delta U
$$

where $\Delta$ denotes the Laplacian in $\mathbb{R}^{3}$. Hence for time harmonic acoustic waves of the form $U(x, t)=\operatorname{Re}\left\{u(x) e^{-i \omega t}\right\}$ with frequency $\omega>0$, the space dependent part $u$ satisfies the Helmholtz equation

$$
\Delta u+k^{2} u=0
$$

in $\mathbb{R}^{3} \backslash \bar{D}$, where the wave number $k>0$ is given by $k^{2}=\omega^{2} / c^{2}$. To describe the phenomenon of scattering mathematically we must distinguish between the two cases of impenetrable and penetrable objects. In particular, for an impenetrable sound soft obstacle the total field $u=u^{i}+u^{s}$, where $u^{i}$ is the incident field and $u^{s}$ is the scattered field, must satisfy the Helmholtz equation in $\mathbb{R}^{3} \backslash \bar{D}$ and the Dirichlet boundary condition $u=0$ on $\partial D$. On the other hand, the scattering by a penetrable inhomogeneous medium $D$ with slowly varying density $\rho_{D}=\rho_{D}(x)$ and sound speed $c_{D}=c_{D}(x)$ differing from the density $\rho$ and sound speed $c$ in the surrounding medium $\mathbb{R}^{3} \backslash \bar{D}$ leads to a transmission problem, i.e., in addition to the scattered field $u^{s}$ in $\mathbb{R}^{3} \backslash \bar{D}$, we have a transmitted wave $v$ satisfying

$$
\Delta v+k^{2} n(x) v=0
$$


in $D$, where $k=\omega / c$ and $n(x)=c^{2} / c_{D}^{2}(x)$ is the index of refraction. The continuity of the pressure and normal velocity across $\partial D$ leads to the transmission conditions

$$
\left.\begin{array}{rl}
u & =v \\
\frac{1}{\rho} \frac{\partial u}{\partial \nu} & =\frac{1}{\rho_{D}} \frac{\partial v}{\partial \nu}
\end{array}\right\} \text { on } \partial D
$$

where $\nu$ is the unit outward normal to $\partial D$. We will always assume for the sake of simplicity that $\partial D$ is of class $C^{2}$, i.e., $\partial D$ can be parameterized by functions that are twice continuously differentiable.

To complete our description of the direct scattering problem we require that the scattered field $u^{s}$ satisfies the Sommerfeld radiation condition

$$
\lim _{r \rightarrow \infty} r\left(\frac{\partial u^{s}}{\partial r}-i k u^{s}\right)=0
$$

where $r=|x|$. Note that of the two possible spherically symmetric solutions $e^{i k r} / r$ and $e^{-i k r} / r$ to the Helmholtz equation, only the first satisfies the radiation condition. Since

$$
\operatorname{Re}\left\{\frac{e^{i k r-i \omega t}}{r}\right\}=\frac{\cos (k r-\omega t)}{r},
$$

this corresponds to an outgoing spherical wave, i.e., the radiation condition characterizes outgoing waves.

Given the above discussion, we can now be more explicit about what we mean by the acoustic inverse scattering problem. In particular, using Green's theorem and the radiation condition it is easy to show (Theorem 2.4 of [13]) that the scattered field $u^{s}$ has the representation

$$
u^{s}(x)=\int_{\partial D}\left\{\left(u^{s}(y) \frac{\partial \Phi(x, y)}{\partial \nu(y)}-\frac{\partial u^{s}}{\partial \nu}(y) \Phi(x, y)\right\} d s(y)\right.
$$

for $x \in \mathbb{R}^{3} \backslash \bar{D}$, where $\Phi$ is the radiating fundamental solution to the Helmholtz equation defined by

$$
\Phi(x, y):=\frac{1}{4 \pi} \frac{e^{i k|x-y|}}{|x-y|}, \quad x \neq y
$$

and $\nu$ again denotes the unit outward normal to $D$. (Here we are of course assuming the existence of a unique solution $u^{s} \in C^{2}\left(\mathbb{R}^{3} \backslash \bar{D}\right) \cap C^{1}\left(\mathbb{R}^{3} \backslash D\right)$ to the direct scattering problem.) Assuming that the incident field is a plane wave moving in the direction $d$, i.e.,

$$
u^{i}(x)=e^{i k x \cdot d}
$$

where $|d|=1$, we see from (1.7) and (1.8) that $u^{s}$ has an asymptotic behavior

$$
u^{s}(x)=\frac{e^{i k r}}{r} u_{\infty}(\hat{x}, d)+O\left(\frac{1}{r^{2}}\right)
$$

as $r \rightarrow \infty$, where $\hat{x}=x /|x|$ and $u_{\infty}$ is the far field pattern of the scattered field $u^{s}$. The inverse scattering problem that we will mainly be concerned with in this paper is 
to determine $D$ from a knowledge of $u_{\infty}(\hat{x}, d)$ for $\hat{x}$ and $d$ on the unit sphere $\Omega:=\{x$ : $|x|=1\}$ and fixed wave number $k$. In the case of a penetrable medium we will also be interested in the possibility of determining the index of refraction $n=n(x), x \in D$. In both cases, we will always assume (except in discussing uniqueness) that $u_{\infty}$ is not known exactly but is determined by measurements which by definition are inexact.

The inverse scattering problem, as defined above, is particularly difficult to solve for two reasons: it is 1) nonlinear and 2) improperly posed. Of these two reasons, it is the latter that creates the most difficulty. In particular, it is easily verified that $u_{\infty}$ is an analytic function of both $\hat{x}$ and $d$ on the unit sphere, and hence, for a given measured far field pattern (i.e., "noisy data"), in general no solution exists to the inverse scattering problem, and, if a solution does exist, it does not depend continuously on the measured data in any reasonable norm. Hence, before we can begin to construct a solution to the inverse scattering problem, we must explain what we mean by a "solution". Motivated by Tikhonov's theory of linear ill-posed problems, in order to determine what we mean by a solution we must introduce "nonstandard" information that reflects the physical situation we are trying to model (e.g., in Tikhonov's theory, such information is used to determine the regularization parameter $\gamma$ in (1.2)). Having resolved the question of what is meant by a solution, we then have to actually construct this solution, and this is complicated not only by the fact that the problem is nonlinear but also by the fact that the above mentioned "nonstandard" information has been incorporated into the mathematical model.

1.2. A Model Inverse Scattering Problem. To fix our ideas, we now consider a simple model problem, a version of which will be considered in more detail in Section 2. In particular, we consider the scattering problem described above where $D$ may now consist of several bounded components, some of which are sound soft and others of which are penetrable (see Figure 1.1). The aim is to determine the support $D$ from a knowledge of the far field pattern $u_{\infty}(\hat{x}, d)$ for $\hat{x}, d \in \Omega$, i.e., $D$ is illuminated by plane waves from every direction $d \in \Omega$ and the resulting scattered field is observed from all directions $\hat{x} \in \Omega$. We call this a "model problem" since in practice $D$ is usually imbedded in a piecewise homogeneous background, i.e., the wave number $k$ is piecewise constant in $\mathbb{R}^{3} \backslash D$, and $d, \hat{x}$ are restricted to a limited aperture. A more realistic problem such as this will be considered in the next subsection, but for now we will outline a numerical procedure for determining $D$ from a knowledge of $u_{\infty}(\hat{x}, d)$ for $\hat{x}, d \in \Omega$. We emphasize that in the above formulation of the inverse scattering problem it is assumed that 1) the number of components of $D$ is unknown and 2) the physical properties of each component are unknown, i.e., it is unknown whether or not a given component is sound soft or penetrable and, if penetrable, what the values of $\rho$ and $\rho_{D}$ are in (1.5).

A method for solving this inverse scattering problem is the linear sampling method originally proposed by Colton and Kirsch [8] and improved by Colton, Piana and Potthast [22] and Kirsch [39], [40]. There are two distinct versions of the linear sampling method, which will be discussed in detail in Sections 4 and 5. To briefly describe these two approaches we first define the far field operator $F: L^{2}(\Omega) \rightarrow L^{2}(\Omega)$ by

$$
(F g)(\hat{x}):=\int_{\Omega} u_{\infty}(\hat{x}, d) g(d) d s(d) .
$$

The first version of the linear sampling method [8], [22] (which can be viewed as a modification of the dual space method of Colton and Monk [13], [16] where the origin 


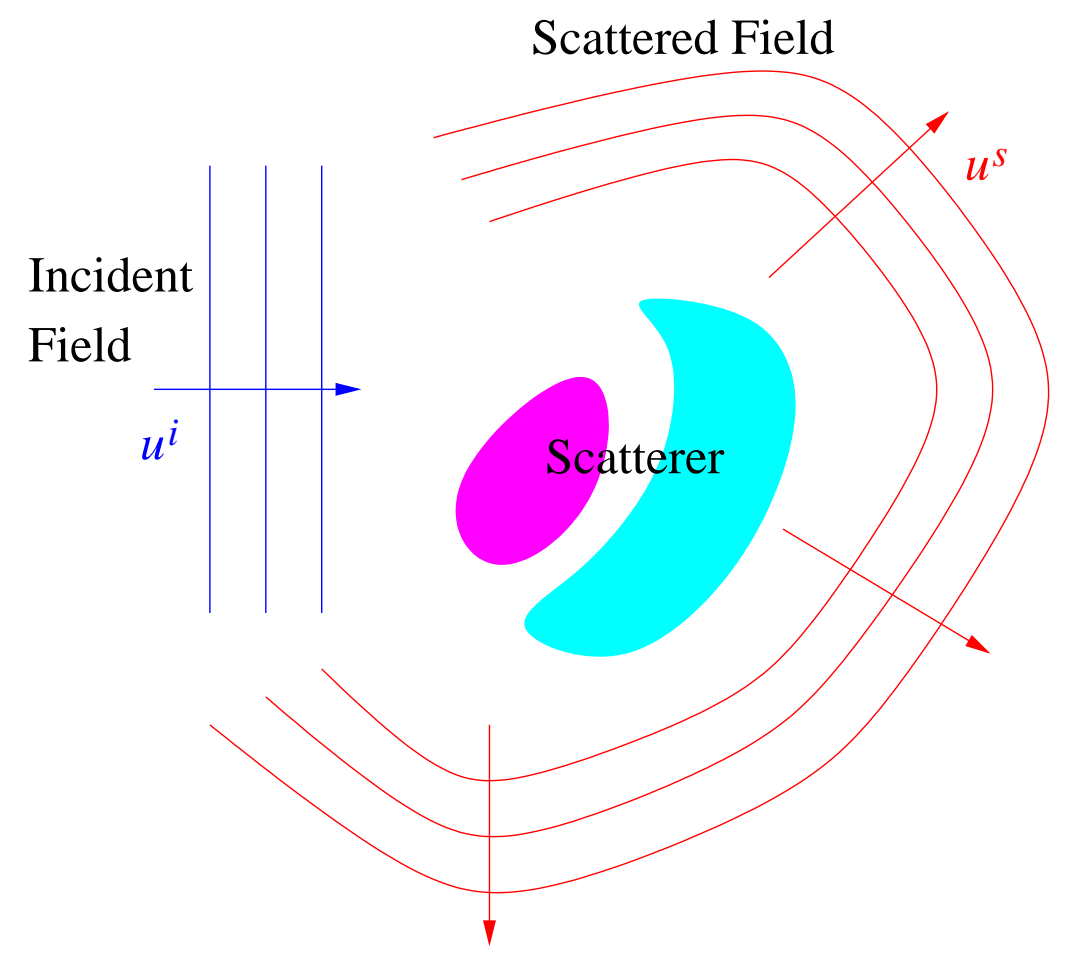

FIG. 1.1. Schematic of the model inverse scattering problem. The incident field is a plane wave (shown in blue) which interacts with the scatterer shown as two colored regions indicating different physical properties. The inverse problem is to determine the support of the scatterer from measurements of the far field pattern of the scattered field due to incident fields from many directions.

is now translated to an arbitrary point $z \in D$ ) then uses regularization methods to solve the linear integral equation

$$
(F g)(\hat{x})=\Phi_{\infty}(\hat{x}, z),
$$

where $\Phi_{\infty}(\hat{x}, z)=\frac{1}{4 \pi} e^{-i k \hat{x} \cdot z}$ is the far field pattern of $\Phi$ as defined by (1.8) and it is assumed that $z \in D$. It can be shown that for every $\epsilon>0$, there exists a function $g=g(\cdot, z) \in L^{2}(\Omega)$ such that $\left\|F g-\Phi_{\infty}\right\|<\epsilon$, and both $\|g(\cdot, z)\|$ and $\left\|v_{g}(\cdot, z)\right\|$ become unbounded as $z$ tends to $\partial D$, where $v_{g}$ is the Herglotz wave function with kernel $g$, defined by

$$
v_{g}(x):=\int_{\Omega} g(d) e^{i k x \cdot d} d s(d)
$$

The unknown boundary $\partial D$ can then be found by solving (1.12) for $z$ on a grid in $\mathbb{R}^{3}$ containing $D$ and looking for those points $z$ where $\|g(\cdot, z)\|$ begins to increase sharply (see Figure 1.2).

A mathematical difficulty with this first version of the linear sampling method is that a characterization of the range of the far field operator is unknown. In particular it is not clear what happens to the behavior of $g$ for $z$ in the exterior of $D$. This difficulty led Kirsch to introduce a modified version of the linear sampling method [39], [40] which is valid for the case of a nonabsorbing medium. In particular, Kirsch 


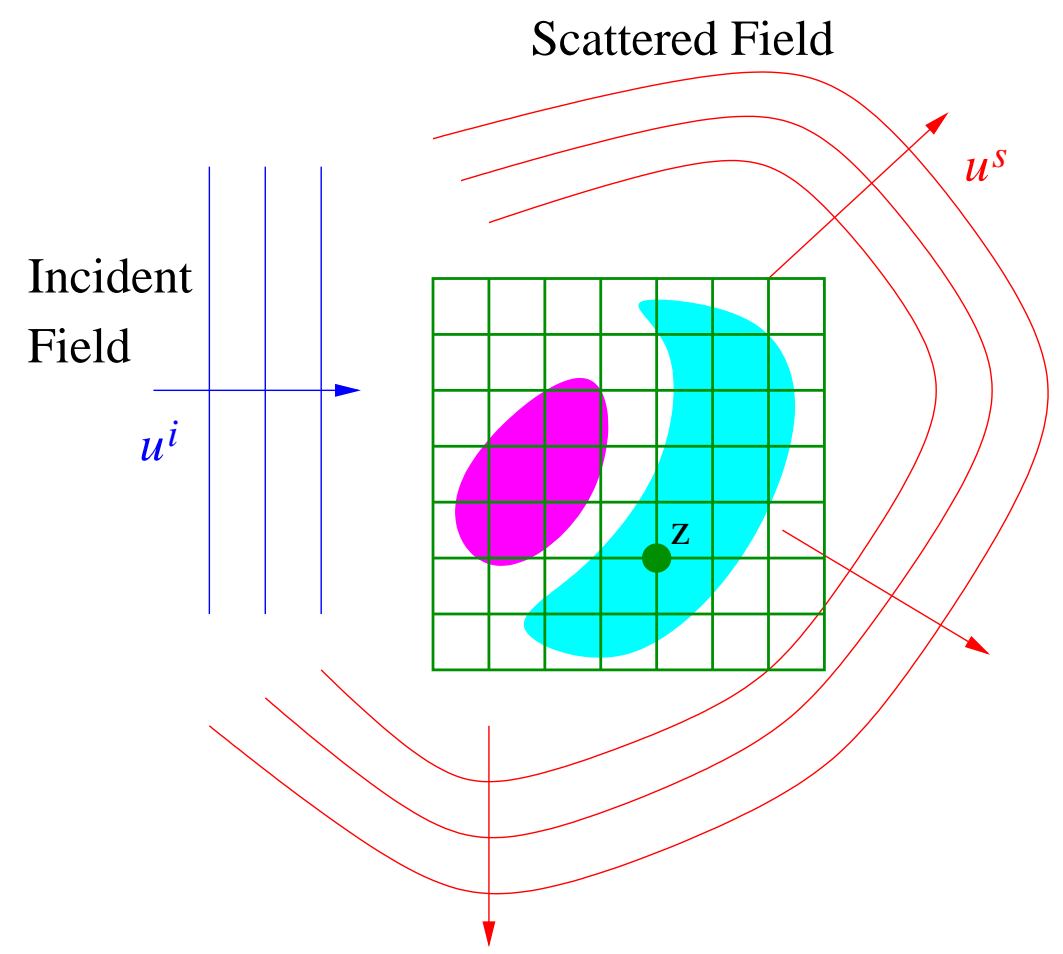

FIG. 1.2. Schematic of the implementation of the linear sampling method for the scattering problem of Figure 1.1. The Herglotz kernel $g(\cdot, z)$ is determined from equation (1.12) for $z$ on a grid containing the scatterer (the grid is shown in green). As $z$ approaches the boundary of the scatterer, $\|g(\cdot, z)\|$ becomes unbounded.

showed that $\Phi_{\infty}(\hat{x}, z)$ is in the range of $\left(F^{*} F\right)^{\frac{1}{4}}$, where $F^{*}$ is the adjoint of $F$ in $L^{2}(\Omega)$, if and only if $z \in D$. A modified linear sampling method then consists of using regularization methods to solve the ill-posed equation $\left(F^{*} F\right)^{\frac{1}{4}} g=\Phi_{\infty}(\cdot, z)$ where $\left(F^{*} F\right)^{\frac{1}{4}}$ is defined by means of a singular system of $F$. As with the original sampling method, $\partial D$ can be found as the locus of points $z$ where $\|g(\cdot, z)\|$ begins to increase sharply. Numerical examples of the implementation of both the original and modified linear sampling method can be found in Section 2.

1.3. The Detection of Buried Objects. As mentioned in the previous section, in most practical situations the unknown scatterer is imbedded in a piecewise homogeneous medium and the directions of incidence and observation are restricted to a limited aperture. An example of such a situation is the detection of buried objects. In particular, consider an object $D$, which may have both sound soft and penetrable components, lying in the lower half space. Assume that the wave number in the lower half space is $k_{2}$ and that the far field pattern of the scattered field is measured in the upper half space which has wave number $k_{1}$ (see Figure 1.3). In this case, if $e_{z}$ is the unit outward normal to the plane bounding the lower half space, the far field pattern $u_{\infty}(\hat{x}, d)$ is only known for $d \in \Omega_{-}:=\left\{x:|x|=1, x \cdot e_{z}<0\right\}$ and $\hat{x} \in \Omega_{+}:=\left\{x:|x|=1, x \cdot e_{z}>0\right\}$, i.e., the scattering data is restricted to a limited aperture. We again assume that 1) the number of components of $D$ is unknown and 2 ) the physical properties of each component of $D$ are unknown. 


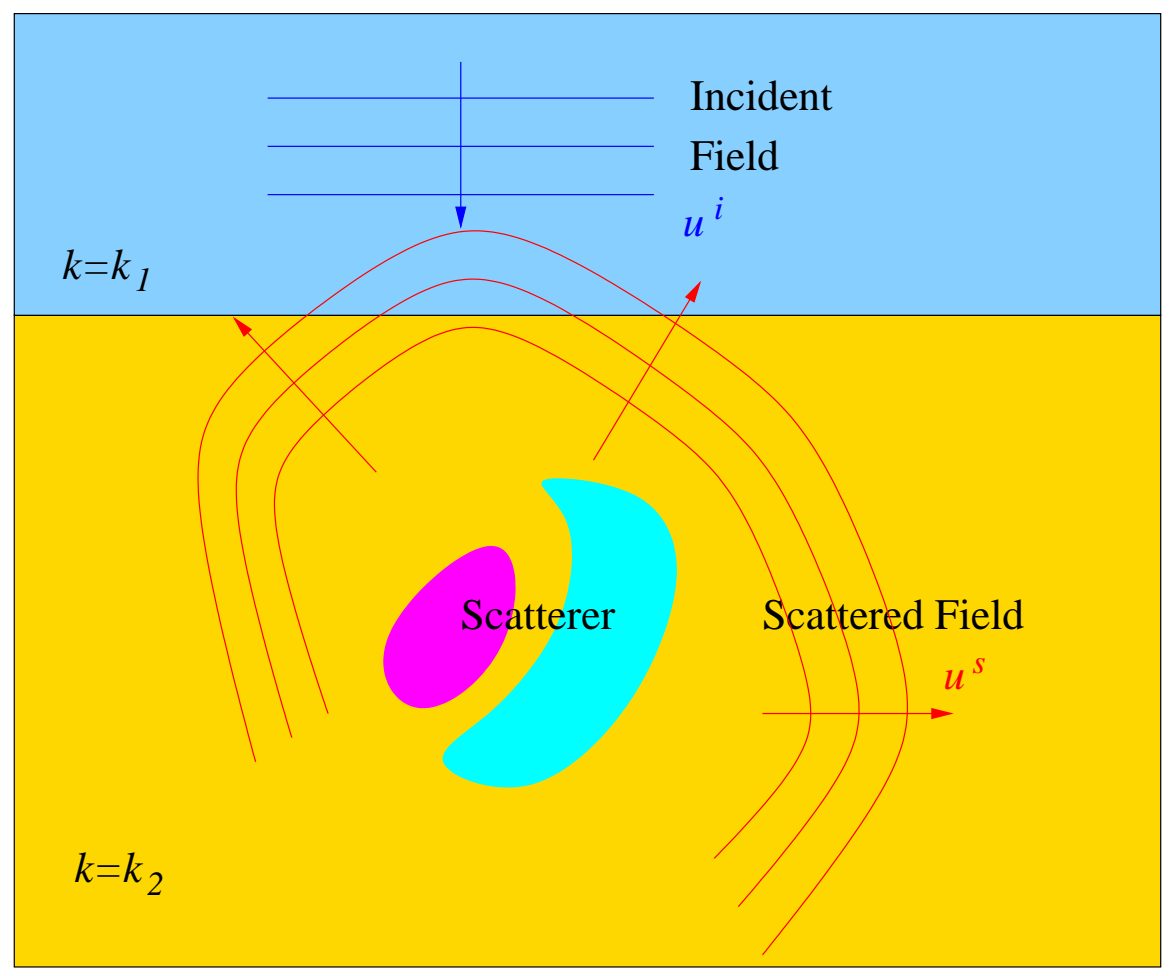

FIG. 1.3. Schematic of the model problem for the detection of buried objects. The incident field is a plane wave from the upper half space (shown in light blue) which interacts with the scatterer buried in the lower half space (shown in yellow). The inverse problem is to determine the support of the scatterer from measurements of the far field pattern of the scattered field in the upper half space.

The above inverse scattering problem can be solved by using a modified version of the linear sampling method. In particular, it can be shown that the conclusions in Section 1.2 remain valid if we replace $F g=\Phi(\cdot, z)$ by the modified far field equation

$$
\int_{\Omega_{-}}\left[u_{\infty}(\hat{x}, d)-u_{b, \infty}(\hat{x}, d)\right] g(d) d s(d)=G_{\infty}(\hat{x}, z), \hat{x} \in \Omega_{+}
$$

where $z \in D, u_{b, \infty}$ is the far field pattern due to scattering by the background medium alone and $G_{\infty}$ is the far field pattern for the Green's function for the background medium. In particular, the solution $g=g(\cdot, z)$ of (1.14) becomes unbounded as $z$ tends to $\partial D$. A related expression is valid if one uses point sources as incident fields and near field data. For mathematical details the reader is referred to [19], [15] and [25]. Numerical examples for a closely related case can be found in Section 3.

1.4. Historical Remarks. The above examples suggest a number of mathematical problems that need to be addressed, in particular the uniqueness of the solution to the inverse scattering problem as well as the mathematical justification of the linear sampling method. These issues will be discussed in Sections 4 and 5 together with brief comments on a number of reconstruction algorithms other than the linear sampling method (which, however, require some knowledge of the physical properties of the scattering object $D$ ). We note that there has also been some effort at establishing 
continuous dependence results based on a priori knowledge of the scattering object [35], [63]. However, at the time of writing, either the a priori assumptions are too stringent or the error estimates are too pessimistic for these results to be useful in practice.

We conclude this Introduction by briefly highlighting the major accomplishments in this mathematical investigation of the inverse scattering problem in preparation for the more detailed discussion in Sections 4 and 5. For the sake of simplicity, in the case of a penetrable medium we will only consider the situation where $\rho=\rho_{D}$ in (1.5).

One of the earliest results in inverse scattering theory was Schiffer's proof that the far field pattern $u_{\infty}(\hat{x}, d)$ for $\hat{x}, d \in \Omega$ uniquely determines the shape of a soundsoft obstacle $D$ [49]. It was subsequently shown by Colton and Sleeman that if it is known a priori that $D$ is contained in a ball of radius $R$ such that $k R<\pi$, then $u_{\infty}(\hat{x}, d)$ for $\hat{x} \in \Omega$, and a single incident direction $d$ uniquely determines $D$ [24]. Unfortunately, as pointed out in [13], Schiffer's proof does not immediately generalize to other boundary conditions. This problem was remedied by Kirsch and Kress who, using an idea originally proposed by Isakov [34], showed that $u_{\infty}(\hat{x}, d)$ for $\hat{x}, d, \in \Omega$ uniquely determines the shape of $D$ as long as the solution of the direct scattering problem depends continuously on the boundary data [42] (see also [13], p. 112 and [39]). In particular, it is not necessary to know the boundary condition a priori in order to guarantee uniqueness!

The first attempt to reconstruct the shape of a sound-soft scattering obstacle from a knowledge of the far field pattern in a manner acknowledging the nonlinear and ill-posed nature of the problem was made by Roger in 1981 [68]. Roger considered the scattering of a plane wave propagating in a fixed direction by a two dimensional sound-soft scatterer parameterized in the form $x=r(\hat{x}) \hat{x}$, where $r(\hat{x})=|x|$, and then solved the nonlinear operator equation $F(r)=u_{\infty}$ by Newton's method, where the Fréchet derivative of $F$ was inverted using Tikhonov regularization. A characterization and rigorous proof of the existence of the Fréchet derivative of $F$ was subsequently established by Kirsch [37] and Potthast [64] (see also [13], [33] and [46]). An alternative approach to solving the inverse scattering problem for a sound-soft obstacle was proposed by Kirsch and Kress [41] (see also [13]), who broke up the inverse scattering problem into two parts. The first part deals with the ill-posedness by constructing the scattered field $u^{s}$ from the far field pattern $u_{\infty}$ by representing $u^{s}$ in the form of a surface potential defined on a surface known a priori to be contained in the unknown scatterer $D$. The second part then deals with the nonlinearity of the problem by determining the unknown boundary of the scatterer as the location of the zeros of the total field $u=u^{i}+u^{s}$ where $u^{i}$ is again a plane wave propagating in a fixed direction. An advantage of this approach is that the cost functional of the nonlinear part of the problem has a particularly simple structure from which the Fréchet derivative is easily computed. Related methods have also been proposed by Angell, Jiang and Kleinman [1], Colton and Monk [16], Misici and Zirilli [52], and Potthast [65], among others.

The nonlinear optimization methods described above have the advantage that only a single incident field is needed for their implementation, e.g. a plane wave propagating in a fixed direction. On the other hand, to use such methods it is necessary to know the number of components of the scatterer as well as a rough idea of the geometry of each component in order to choose an appropriate parameterization of the surface. In addition, it is also necessary to know the boundary condition satisfied by the field on the surface of the scatterer, i.e. whether it is sound-soft or not. The 
linear sampling method, discussed above, avoids these problems and will be examined in detail in Sections 4 and 5.

We now turn to the problem of reconstructing the index of refraction from a knowledge of the far field pattern, i.e., the direct scattering problem is (1.3)-(1.5) where $u=u^{i}+u^{s}$ and we wish to determine $n=n(x)$ from a knowledge of $u_{\infty}(\hat{x}, d)$ when $u^{i}(x)=e^{i k x \cdot d}$. Although most of the research in this area has made the assumption (possibly motivated by the case of quantum mechanics) that $n \in C^{1}\left(\mathbb{R}^{3}\right)$, with $n(x)=1$ for $x \in \mathbb{R}^{3} \backslash D$, we shall make the physically more reasonable assumption that $n$ is continuously differentiable in $\bar{D}$ with $n(x)=1$ for $x \in \mathbb{R}^{3} \backslash \bar{D}$ but $n(x) \neq 1$ for $x \in \partial D$, i.e. $n$ has a jump discontinuity across the $C^{2}$ boundary $\partial D$ and $\rho$ is not necessarily equal to $\rho_{D}$. As already mentioned, for the sake of simplicity, we shall only consider the special case $\rho=\rho_{D}$. Modifications for the case $\rho \neq \rho_{D}$ will be indicated in Section 5.

As with obstacle scattering, the first issue of concern is that of uniqueness, i.e., does the far field pattern $u_{\infty}(\hat{x}, d)$ for $\hat{x}, d \in \Omega$ and fixed wave number $k$ uniquely determine the index of refraction $n=n(x)$ ? Based on the fundamental work of Sylvester and Uhlmann [71], this question was answered in the affirmative by Nachman [54], Novikov [57] and Ramm [66] in 1988 (see also [67]). The key step in the proof was to show that products $v_{1} v_{2}$ of solutions to $\Delta v_{1}+k^{2} n_{1} v_{1}=0$ and $\Delta v_{2}+k^{2} n_{2} v_{2}=0$ for two different refractive indices $n_{1}$ and $n_{2}$ are complete in $L^{2}(D)$ for any bounded domain $D \subset \mathbb{R}^{3}$. Such a result was in turn obtained by constructing special solutions of $\Delta v+k^{2} n v=0$ that behave asymptotically like $e^{i z \cdot x}$, where $z \in \mathbb{C}^{3}$, the space of three complex variables. The original technically difficult construction of these special solutions using Fourier integral techniques has recently been considerably simplified by Hähner through the use of Fourier series [32].

All existing methods for determining the index of refraction from noisy far field data without linearizing the problem are based on nonlinear optimization methods. The simplest of these is obtained by using Green's formula (cf. Theorem 2.1 of [13]) to rewrite the scattering problem (1.3)-(1.5) (for $\rho=\rho_{D}$ ) as the Lippmann-Schwinger equation

$$
u(x)=e^{i k x \cdot d}-k^{2} \iint_{\mathbb{R}^{3}} \Phi(x, y) m(y) u(y) d y, x \in \mathbb{R}^{3},
$$

where $\Phi$ is defined by (1.8) and $m:=1-n$. From (1.15) it is easily deduced that

$$
u_{\infty}(\hat{x}, d)=-\frac{k^{2}}{4 \pi} \iint_{\mathbb{R}^{3}} e^{-i k \hat{x} \cdot y} m(y) u(y) d y
$$

where $u(y)=u(y, d)$. Assuming $u_{\infty}(\hat{x}, d)$ is known for $\hat{x} \in \Omega$ and for $p$ incident waves $d=d_{1}, d_{2}, \ldots, d_{p}$, a nonlinear optimization scheme for determining $m$ and $u\left(y, d_{j}\right), j=1,2, \ldots, p$, is easily formulated from (1.15) and (1.16). Due to the simple manner in which $m$ and $u$ appear in (1.15), (1.16), the Fréchet derivative is easily computed. One can also, of course, work directly with the scattering problem (1.3)-(1.5) instead of reformulating it as the Lippmann-Schwinger integral equation. Examples of successful numerical reconstructions using such methods have been given by Gutman and Klibanov [29], Kleinman and van den Berg [43], Natterer and Wübbeling [55], Tabbara et al.[73], and Wang and Chew [77], among others. As with obstacle scattering, because of the ill-posed nature of the inverse scattering problem, regularization methods must be used to compute the solution. 
An alternative method to that discussed above for determining the index of refraction from noisy far field data has been proposed by Colton and Monk [13], [17]. This method resembles that of Kirsch and Kress in obstacle scattering in that the problem is broken up into a linear ill-posed part and a nonlinear optimization part, and it has the advantage over the methods described above of being able to increase the number of incident fields without increasing the cost of solving the inverse problem. This method will be discussed in more detail in Section 5 .

Unfortunately, the computation of $n=n(x)$ through the use of nonlinear optimization methods is extremely time consuming for realistic three dimensional situations. However, in many cases of practical interest, a complete determination of the index of refraction is far more than is needed. It is often sufficient to determine the number of objects present and the support of each of them. Such information is provided by the linear sampling method as described above and, in more detail, in Sections 5 and 6. Occasionally even less information can be useful, for example a lower bound for the volume of the scatterer. In special cases such a lower bound can be obtained from a knowledge of the spectrum of the far field operator $F$ defined by (1.11). In particular, if the index of refraction of the unknown scatterer is known to be constant with positive imaginary part, then the eigenvalues of $F$ are all contained inside the circle $|\lambda|^{2}-\frac{4 \pi}{k} \operatorname{lm} \lambda=0$ in the complex $\lambda$ plane, and a knowledge of the radius of the smallest circle with center on the axis $\operatorname{Re} \lambda=0$ and passing through the origin that contains all the eigenvalues yields a lower bound to the volume of the scatterer [12].

In concluding this Introduction, we would like to emphasize that most of the above results for acoustic waves have analogues for electromagnetic waves and we refer the reader to the monograph [13] and the paper [23] for details and further references. We also make no claim to cover all the many topics in inverse scattering theory for acoustic waves. Indeed, with the rapid growth of the field, such a task would be impossible in a single survey paper. Instead, we have been motivated by our own view of inverse scattering which focuses on the issues of uniqueness and numerical reconstructions. In particular, the emphasis of this survey is on the research done at the Universities of Delaware, Göttingen and Karlsruhe during the past fifteen years. Nevertheless, we feel that we have succeeded in presenting some of the highlights of the mathematical and numerical foundations of time harmonic acoustic inverse scattering theory and hope that our effort will encourage others to enter this exciting field of applied mathematical research.

In addition to the research program at Delaware, Göttingen and Karlsruhe, there are of course many other groups in inverse scattering with their own research agendas. We mention in particular the considerable effort that has been made in nonlinear iteration techniques by Gutman and Klibanov [29], Kleinman and van den Berg [43], Natterer and Wübbeling [55] and Wang and Chew [77], in layer striping by Chen and Rokhlin [4] and Sylvester and Winebrenner [72] and diffraction tomography by Devaney [28] and Langenberg [48] (see also the references in [13]).

2. A Model Problem Using Real Data. We now return to the model problem considered in Section 1.2 of the Introduction. Under appropriate assumptions, the time-harmonic electromagnetic direct scattering problem for an infinite cylinder can 
be reformulated as the problem of determining $u$ from the equations

$$
\begin{gathered}
\triangle u+k^{2} u=0 \quad \text { in } \mathbb{R}^{2} \backslash \bar{D}, \\
u=u^{i}+u^{s}, \\
\lim _{r \rightarrow \infty} \sqrt{r}\left(\frac{\partial u^{s}}{\partial r}-i k u^{s}\right)=0
\end{gathered}
$$

where $D$ is the cross-section of the cylinder and $u$ satisfies certain boundary conditions on $\partial D$. More specifically, when the cylinder is impenetrable $u$ satisfies the Dirichlet boundary condition $u=0$ on $\partial D$ and when $D$ is a penetrable inhomogeneous medium the transmission boundary conditions (1.5) are satisfied for $\rho=\rho_{D}$ and $x \in \mathbb{R}^{2}$. In particular, the problem we are concerned with is the two-dimensional analogue of that considered in Section 1.2. Our aim is to use the numerical procedure suggested by the discussion in Section 1.2 for determining $\partial D$ from noisy far field data, delaying a mathematical justification of this procedure until Sections 4 and 5 of this paper. Recall that the idea is to sample a region (by varying $z$ ) where the unknown object is thought to be and plot $\|\varphi(\cdot, z)\|$ where $\varphi$ is the numerical approximation to $g \in L^{2}(\Omega)$ and $g$ satisfies either

$$
\left\|F g-\Phi_{\infty}(\cdot, z)\right\|<\epsilon
$$

or

$$
\left\|\left(F^{*} F\right)^{\frac{1}{4}} g-\Phi_{\infty}(\cdot, z)\right\|<\epsilon
$$

depending on whether the first or second version of the linear sampling method is used.

To determine $\varphi$, which minimizes the Tikhonov functional (1.2) for $A$ being either $F$ or $\left(F^{*} F\right)^{\frac{1}{4}}$, it is sufficient to solve the normal equation

$$
\gamma \varphi+A^{*} A \varphi=A^{*} \Phi_{\infty}(\cdot, z) .
$$

Hence, once $\gamma>0$ is chosen, using the singular value decomposition $F=U S V^{*}$, we have from (2.1) that

$$
\|\varphi\|^{2}=\sum_{i=1}^{N}\left(\frac{s_{i}}{s_{i}^{2}+\gamma}\right)^{2}\left|U^{*} \Phi_{\infty}(\cdot, z)\right|^{2}
$$

and

$$
\|\varphi\|^{2}=\sum_{i=1}^{N} \frac{s_{i}}{\left(s_{i}^{2}+\gamma\right)^{2}}\left|V^{*} \Phi_{\infty}(\cdot, z)\right|^{2}
$$

for $A=F$ and $A=\left(F^{*} F\right)^{\frac{1}{4}}$, respectively, where $\left\{s_{i}\right\}$ are the singular values of $F$.

The parameter $\gamma$ is chosen by Morozov's generalized discrepancy principle [75] and, as a result of the singular value decomposition, is the zero of the monotonically increasing functions

$$
f(\gamma)=\sum_{i=1}^{N} \frac{\gamma^{2}-\delta^{2} s_{i}^{2}}{\left(s_{i}^{2}+\gamma\right)^{2}}\left|U^{*} \Phi_{\infty}(\cdot, z)\right|^{2}
$$




$$
f(\gamma)=\sum_{i=1}^{N} \frac{\gamma^{2}-\delta^{2} s_{i}}{\left(s_{i}+\gamma\right)^{2}}\left|V^{*} \Phi_{\infty}(\cdot, z)\right|^{2}
$$

for the first and second versions respectively. The parameter $\delta$ is chosen such that $\left\|F-F_{\delta}\right\|<\delta$, i.e., $\delta$ is an estimate of the noise level. To summarize, for a given estimate $\delta, \gamma$ is determined by solving $f(\gamma)=0$ and then $\|\varphi\|$ is computed using (2.2) or (2.3) respectively.

To demonstrate the capabilities of the linear sampling method, we now provide results using the method on real data. The data is the Ipswich data provided by the Electromagnetics Technology Division at Hanscom Air Force Base. The Ipswich data is single frequency electric far field data measured using a multi-static system with multiple views corresponding to different incident angles. In this case, it is not possible to measure scattering at or near backscattering directions since the receiver and transmitter cannot be physically coincident. As a result, the Ipswich data does not provide a "full" view of the target for each incident angle. A more detailed discussion of the data and the measurement process can be found in [51].

We consider two targets: Ips009 - an aluminum triangle and Ips010 - a plexiglas triangle. The data is given for TM mode electromagnetic waves with a frequency of $10 \mathrm{Ghz}$ (corresponding to a wavelength $\lambda=3 \mathrm{~cm}$ ). In each case, there are 36 different incident angles ranging from 0 to 350 degrees in increments of 10 degrees and, for each incident angle, there are 18 observation angles given by

$$
\theta_{o}=\theta_{i}+180+10 n \text { for } n=0, . ., 17
$$

where $\theta_{i}$ is the incident angle and $\theta_{o}$ is the observation angle. The data only partially fills in the discretized far field operator (the matrix corresponding to $F$ ) but, using reciprocity, almost all of the missing entries of this matrix can be determined. The remaining unknown entries, which correspond to backscattering measurements, are approximated by averaging the two adjacent known measurements for the same incident angle.

The only a priori information known about the location of the obstacles is that the minimum circumscribing circle centered at the origin has a radius of $6 \mathrm{~cm}$, so a square sampling grid ( $40 \times 40$ or 1600 sampling points) is taken on a square with side $14 \mathrm{~cm}$ centered at the origin. The results of using (2.2) and (2.3) are shown in Figures 2.1 and 2.2 respectively. In each case $\|\varphi\|^{-1}$ is plotted for the Morozov parameter $\delta$ chosen to be 0.22 . This value of $\delta$ yields the best reconstruction from those values considered by us.

We note that the reconstruction of the (penetrable) plexiglas triangle is considerably poorer than that of the (impenetrable) aluminum triangle. This is also the case when either weak scattering techniques or nonlinear optimization methods are used to reconstruct the same objects [61], [76] and hence is not a problem associated specifically with the linear sampling method.

3. Numerical Experiments for the Detection of Buried Objects. We shall now present some numerical results illustrating the use of the linear sampling method to detect the location and shape of buried objects as described in Section 1.3 (but now using near field data). We start by discussing the forward problem, then proceed to a method for detecting the support of buried objects motivated by the results mentioned in Section 1.3, and finally we describe the numerical results. 


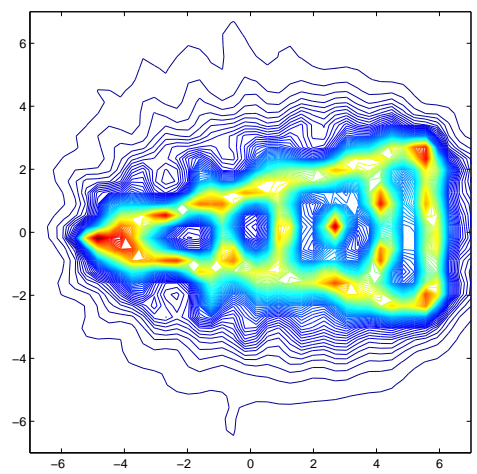

(a) Ips009 using $F$

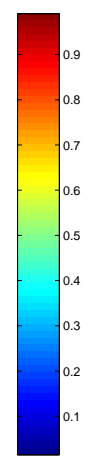

FIG. 2.1. Plot of $\|\varphi\|^{-1}$ for an aluminum triangle (target Ips009): (a) using the linear sampling method associated with $F$ and $(b)$ using the linear sampling method associated with $\left(F^{*} F\right)^{\frac{1}{4}}$.

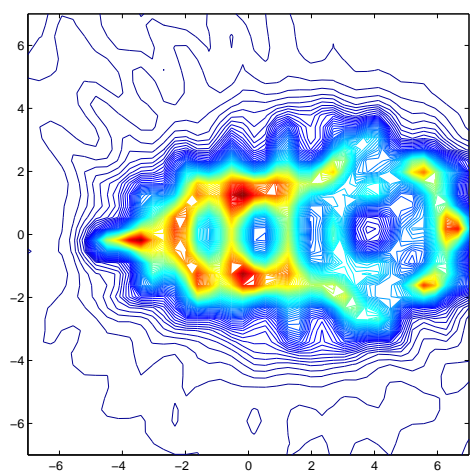

(a) Ips010 using $F$

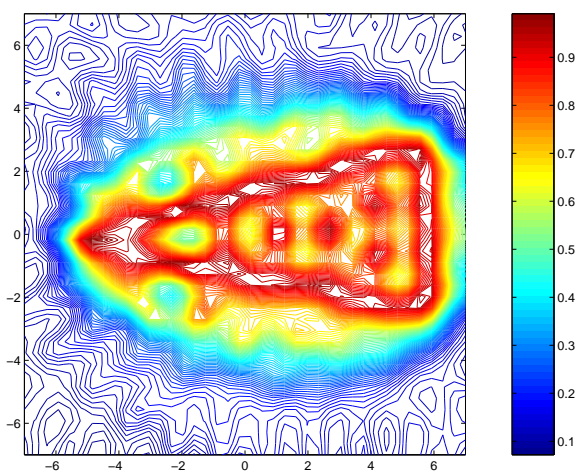

(b) Ips009 using $\left(F^{*} F\right)^{\frac{1}{4}}$

FIG. 2.2. The same plot as Figure 2.1 but for a plexiglass triangle (target Ips010).

We suppose that the background medium consists of two homogeneous, isotropic half spaces meeting at $x_{3}=0$. One can think of this as a simplified model of water over a flat sand layer. The buried object is represented by a bounded region $D$ strictly contained in the lower half space (i.e. $\bar{D} \subset\left\{x \in \mathbb{R}^{3} \mid x_{3}<0\right\}$ ). Acoustic waves are excited in this system by a point source located at $x=y$ in the upper half space (i.e. $y_{3}>0$ ) see Figure 3.1. Under appropriate assumptions, the acoustic velocity potential $u=u(x)$ satisfies the Helmholtz equation in each half space. We denote by $u_{i}, i=1,2$ the field in the upper and lower half space respectively. Then

$$
\begin{aligned}
& \Delta u_{1}+k_{1}^{2} u_{1}=\delta_{y} \text { for } x \in \mathbb{R}^{3}, \quad x_{3}>0, \\
& \Delta u_{2}+k_{2}^{2} u_{2}=0 \text { for } x \in \mathbb{R}^{3} \backslash \bar{D}, \quad x_{3}<0 .
\end{aligned}
$$

Here $k_{1}$ and $k_{2}$ are the wave numbers for the materials in the upper and lower half space and $\delta_{y}$ is the delta function at the point $y$. We shall actually use wave numbers appropriate for water and sand [3], in particular $k_{1}=3.6, k_{2}=4$.

Across the interface between the layers the pressure field is continuous but the 


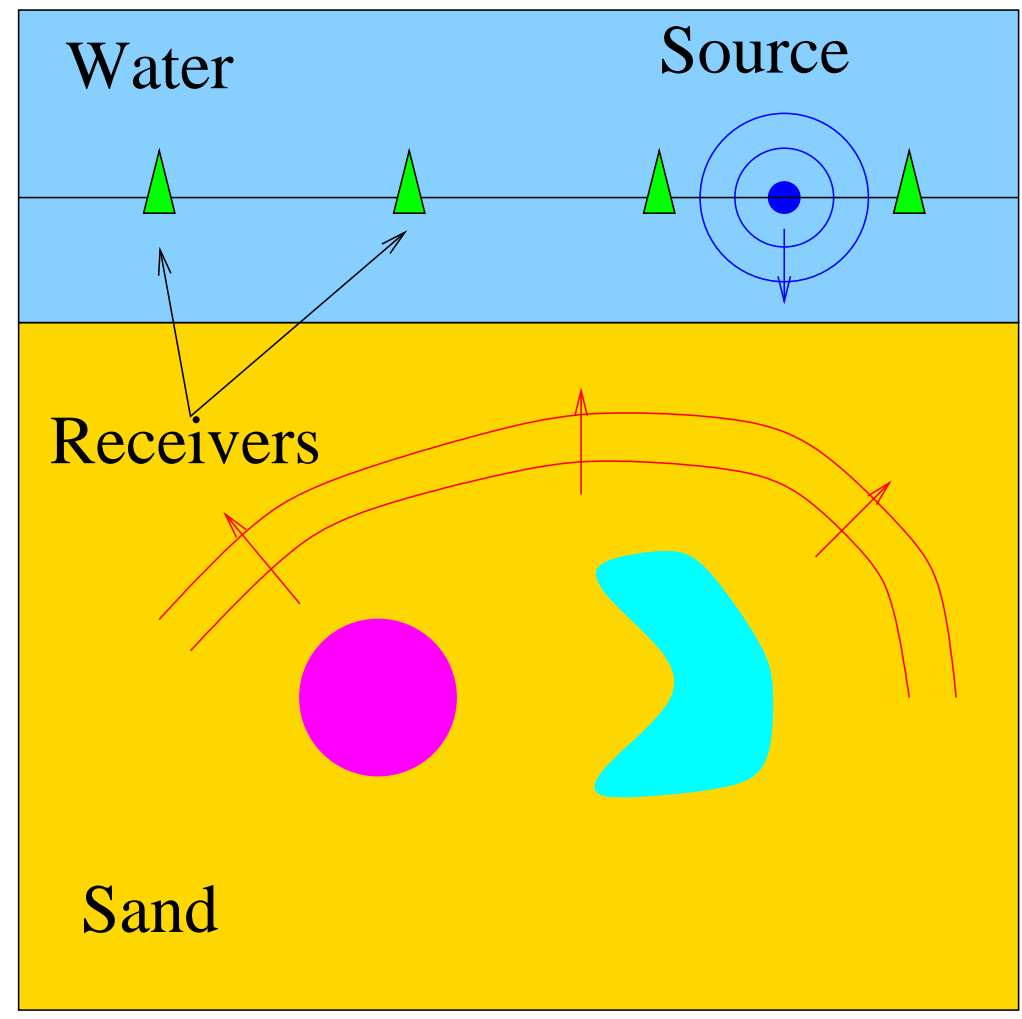

FIG. 3.1. Schematic of the inverse scattering problem for buried objects. The incident field is due to a source located in the water (shown in blue) which interacts with the scatterer shown as two colored regions indicating different physical properties (in the sand layer). The inverse problem is to determine the support of the scatterer from measurements of the scattered field in the water due to incident fields from many sources.

flux can jump (due to changes in density as in (1.5)). Thus, for some $\alpha>0$ we have

$$
u_{1}=u_{2}, \quad \frac{\partial u_{1}}{\partial x_{3}}=\alpha \frac{\partial u_{2}}{\partial x_{3}} \text { for } x \in \mathbb{R}^{3}, \quad x_{3}=0 .
$$

For the case of water and sand an appropriate choice of $\alpha$ is $\alpha=1 / 2[3]$.

We shall restrict ourselves to a sound soft scatterer, and assume that

$$
u=0 \text { on } \partial D \text {. }
$$

Finally, we need a criterion to obtain a unique solution to this scattering problem, and to this end we use the integral radiation condition due to Odeh [59],

$$
\int_{\Sigma_{R}}\left|\frac{\partial u}{\partial r}-i k u\right|^{2} d s \rightarrow 0
$$

as $R \rightarrow 0$ where

$$
\Sigma_{R}=\left\{x \in \mathbb{R}^{3}|| x \mid=R\right\}
$$

and $k=k_{1}$ in the upper half space and $k=k_{2}$ in the lower half space. 
Using Odeh's argument, it is possible to show that there exists at most one classical solution to (3.1)-(3.5). Concerning the existence of a solution, Coyle and Monk [27] have given a variational proof of existence for a more general layered scattering problem in $\mathbb{R}^{2}$, and these methods can also be used in $\mathbb{R}^{3}$.

The forward problem is thus to compute $u_{1}$ and $u_{2}$ satisfying (3.1)-(3.5) given $D, k_{1}, k_{2}, \alpha$ and $y$. For our numerical experiments, these fields are computed via a finite element method [26]. Let $u(x, y)$ denote the solution of this problem at position $x$ due to the source at position $y$.

The inverse problem also assumes a knowledge of $k_{1}, k_{2}$ and $\alpha$. But now we wish to find $D$ given a knowledge of $u_{1}$ for a collection of source points $y$ located in the upper half space. More precisely, we assume that there is a rectangle $R \subset \mathbb{R}^{3}$ such that we know $u(x, y)$ for all $x \in R$ and all $y \in R$. In our numerical experiments we choose

$$
\begin{gathered}
R=\left\{\left(x_{1}, x_{2}, x_{3}\right) \mid, x_{\min } \leq x_{1} \leq x_{\max }, y_{\min } \leq x_{2} \leq y_{\max }\right. \\
\left.x_{3}=z_{\min }>0\right\}
\end{gathered}
$$

where $x_{\min }, y_{\min }, x_{\max }, y_{\max }$ and $z_{\min }$ are chosen depending on the numerical experiment we wish to simulate. Since the rectangle $R$ is of limited extent, the data for the inverse problem is available over a limited aperture, which implies that the solution of the inverse problem will be degraded compared to situations in which data can be gathered on a sphere containing the object.

We shall use the linear sampling method to approximate $\partial D$. In order to do this we need the Green's function for the background layered medium (i.e., $D$ is absent). We denote the Green's function by $G(x, y)$. This is computed using Sommerfeld's technique [70] and details can be found in [26]. Since we make the assumption that $D$ is completely buried, we do not need to evaluate $G(x, y)$ when $x$ or $y$ are on the interface between the layers. Thus the integrals representing $G$ converge rapidly and we do not have difficulties with slowly decaying tails of the integrals as can happen when $x$ and $y$ are on the interface.

In setting up the linear sampling method it is convenient to separate the field $u$ into an incident field

$$
u^{i}(x)=G(x, y)
$$

and a resulting scattered field, so that

$$
u=u^{i}+u^{s} \text { in } \mathbb{R}^{3} \backslash D .
$$

Note that $u^{i}$ is the solution of the layered medium problem in the absence of $D$. The linear sampling method is then based on finding $g_{z}$ such that

$$
\int_{R} u^{s}(x, y) g_{z}(y) d s(y)=G(x, z), \forall x \in R
$$

for various points $z$ in the lower half space. (Note that this is equivalent to the integral equation (1.14) for the case of near field data, i.e. instead of using the kernel $u^{s}-u_{b}^{s}$, where $u^{s}$ and $u_{b}^{s}$ are the scattered fields due to point sources, we use the scattered field $u^{s}$ corresponding to the Green's function as incident field (see Section 5).) In particular, it can be shown (see [25] for the case in $\mathbb{R}^{2}$ ) that there exists an approximate solution $\varphi_{z}$ to (3.6) such that $\left\|\varphi_{z}\right\| \rightarrow \infty$ as $z$ approaches $\partial D$ for 
$z \in D$. To derive a numerical method we shall approximate the solution of the above integral equation using Tikhonov regularization and Morozov's discrepancy principle for $z$ lying on a grid in $\mathbb{R}^{3}$ in the region of interest (the region where we wish to probe to find if a scatterer is present). We then plot the iso-surfaces of $1 /\left\|\varphi_{z}\right\|$ which is the surface consisting of all points $z$ such that $1 /\left\|\varphi_{z}\right\|=C$ where $C$ is chosen close to zero (we shall say more about this choice shortly) and this is our prediction of $\partial D$.

In our numerical experiments we discretize (3.6) using the trapezoidal rule so that $x$ and $y$ lie at uniformly spaced lattice points on $R$ (this corresponds to making measurements of $u$ for a finite number of source positions $y$ and receiver positions $x$ ). In this paper we shall show results for a $21 \times 21$ lattice of values on $R$. The probe point $z$ is also varied on a uniform lattice in a box known to contain $D$ (a more efficient adaptive approach is given in [6]).

In practice it is difficult to know when $1 /\left\|\varphi_{z}\right\| \approx 0$ since $\varphi_{z}$ is computed from noisy data using the Morozov technique mentioned above. We have found the "calibration" approach of [6] is a usable heuristic. In this technique we use the desired source and receiver combination and background to solve for the scattered field from a known object of similar size to our intended target. We can then choose a value of $C$ such that the surface $1 /\left\|\varphi_{z}\right\|=C$ is a good approximation of the known scatterer. Using this value of $C$ we can then use the surface $1 /\left\|\varphi_{z}\right\|=C$ as a prediction of $\partial D$ for the unknown scatterer.

In the numerical experiments, we first choose a scatterer and source-receiver combination and then use the finite element method to predict an approximation to $u(x, y), x, y \in R$. Then to avoid any possibility of "inverse crimes" (these are unrealistically good reconstructions resulting from interactions between the numerical schemes for the forward and inverse problem, see [13]), we corrupt the field $u$ computed by finite elements with noise and define $\tilde{u}$ by

$$
\tilde{u}(x, y)=u(x, y)\left(1+\epsilon \chi(x, y)+i \epsilon \chi_{1}(x, y)\right),
$$

where $\chi$ and $\chi_{1}$ are normally distributed random numbers in $[-1,1]$ and $\epsilon$ is an error parameter. Discretizing (3.6) using the trapezoidal rule and putting $\tilde{u}$ in place of $u$ results in a matrix equation to be solved for each $z$. The field $\tilde{u}$ is then used in the Tikhonov/Morozov algorithm, and the error parameter in this technique is the spectral norm of the difference between the matrices corresponding to the finite element approximation of $u$ and $\tilde{u}$. This does not include the error due to the finite element method, which could be much larger than the error due to the artificial random noise added in (3.7).

All our examples are smaller than a wavelength in size. This makes an accurate reconstruction difficult. In the figures, the wavelength in each layer is indicated by horizontal red lines. The sources/receivers rectangle $R$ is denoted by a blue waffle pattern. The brown rectangle shows the position of the interface and the green parallelepiped shows the region in which $z$ is varied. The predicted scatterer is shown in red.

In Figure 3.2 we show the results of running our algorithm on a single spherical scatterer. First data is generated by a forward finite element code [26], then it is corrupted as in (3.7) using $\epsilon=0.07$. This gives a relative error for the matrix corresponding to the kernel of (3.6) of roughly $0.5 \%$ in the spectral norm (this corresponds to an error of roughly $9 \%$ with respect to the matrix maximum norm). The measurement region is located at $z_{\min }=1$ and $x_{\min }=y_{\min }=-1.5, x_{\max }=y_{\max }=1.5$ and there are 21 data points in each direction. Although this may appear to be a large 


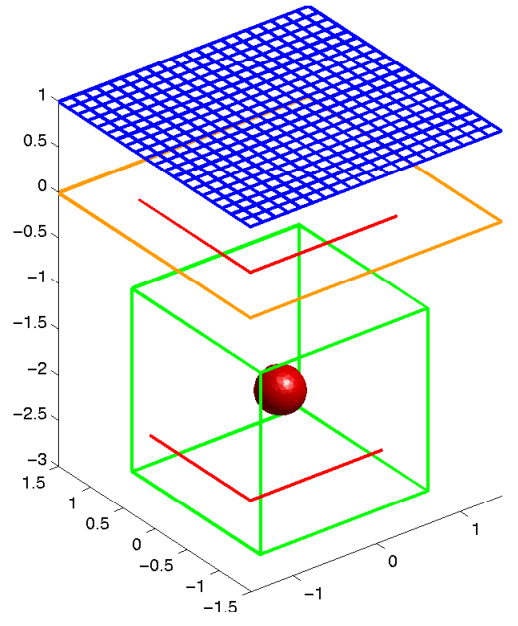

(a) Exact scatterer (3D view)

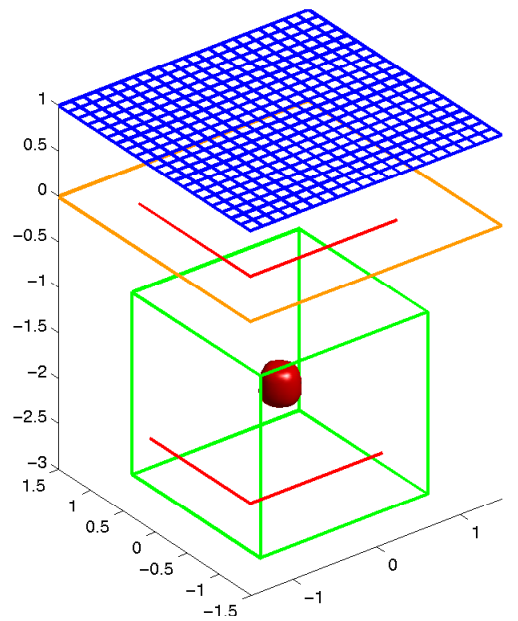

(c) Reconstructed scatterer (3D view)

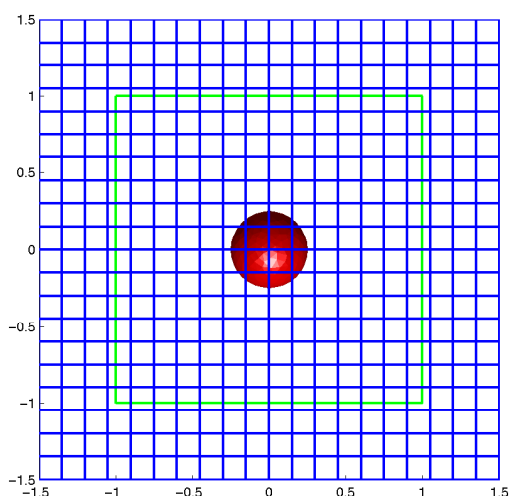

(b) Exact scatterer (view down $x_{3}$ axis)

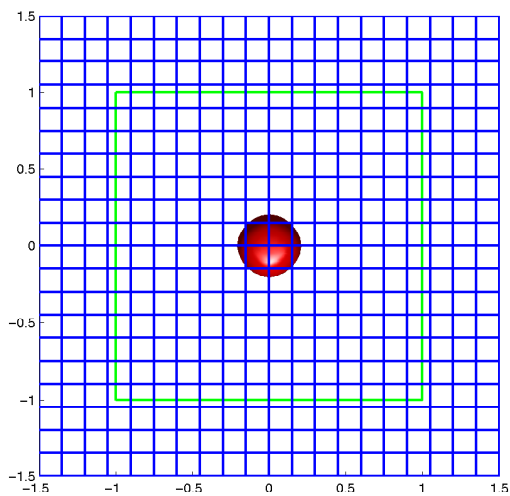

(d) Reconstructed scatterer (view down $x_{3}$ axis)

FIG. 3.2. Reconstruction of a sphere using the isovalue $1 / 3$ when $\epsilon=0.07$. This example is used for calibration purposes. The value of $1 / 3$ is chosen by eye to give a reasonable reconstruction of the sphere in the horizontal and vertical directions. In all the figures the wavelength in each layer is indicated by horizontal red lines. The sources/receivers rectangle $R$ is denoted by a blue waffle pattern. The brown rectangle shows the position of the interface, and the green parallelepiped shows the region in which $z$ is varied. The exact or predicted scatterer is shown in red.

amount of data, the aperture (when viewed from the sphere) is only 50 degrees and so is close to the minimum found acceptable by Colton and Piana [21]. This minimum also seems to apply when other reconstruction techniques are used [58], [79]. By normalizing the maximum value of $1 /\left\|\varphi_{z}\right\|$ to one and viewing a variety of iso-surfaces we find that $1 /\left\|\varphi_{z}\right\|=1 / 3$ gives an acceptable reconstruction and we shall use this value for all reconstructions using this measurement array and problem parameters.

In Figure 3.3 we show the results of reconstructing a pair of spherical scatterers 


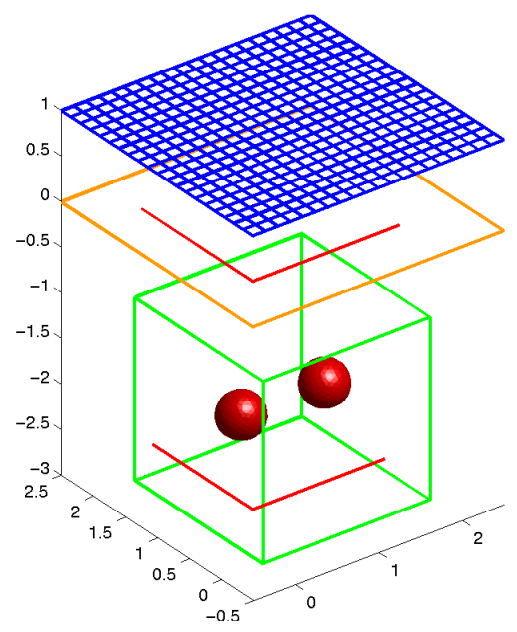

(a) Exact scatterer (3D view)

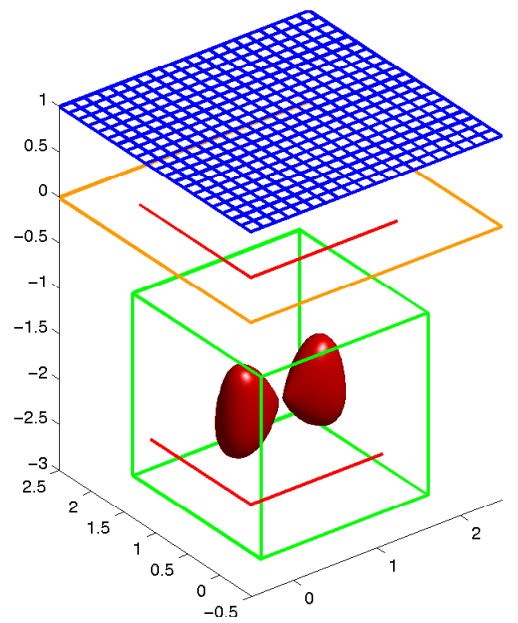

(c) Reconstructed scatterer (3D view)

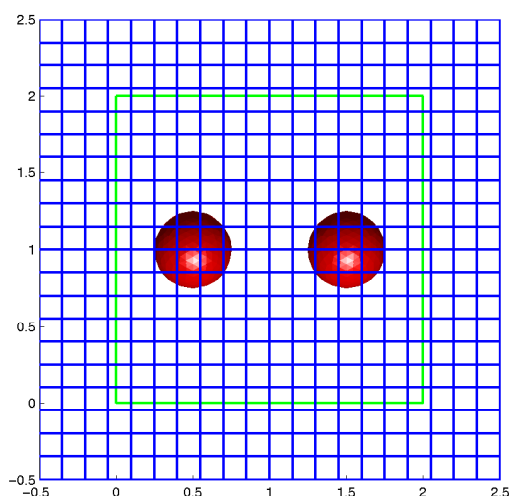

(b) Exact scatterer (view down $x_{3}$ axis)

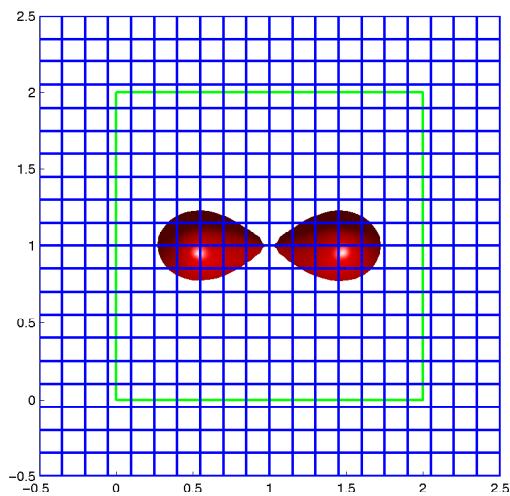

(d) Reconstructed scatterer (view down $x_{3}$ axis)

FIG. 3.3. Reconstruction of two spheres using the same measurement array and parameters as for Figure 3.2. In keeping with the notion of calibration we choose the isovalue for the display of the numerical reconstruction to be $1 / 3$.

using the same measurement array and parameters as for Figure 3.2. In keeping with the "calibration" philosophy we show the iso-surfaces for $1 /\left\|\varphi_{z}\right\|=1 / 3$.

Our final figure is an L-shaped scatterer gridded using the QMG mesh generator [53]. In this case we did not try to capture the underside of the L. Results are shown in Figure 3.4.

As the aperture decreases or the noise level increases the quality of the reconstruction deteriorates. For example, using double the noise (i.e., $\epsilon=0.14$ or about $1.1 \%$ spectral norm error) results in a somewhat higher calibration isovalue than for the lower noise case shown previously. The results are now shown in Figure 3.5 (we 


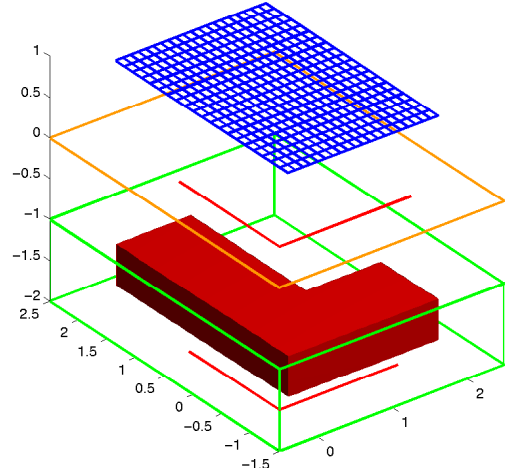

(a) Exact scatterer (3D view).

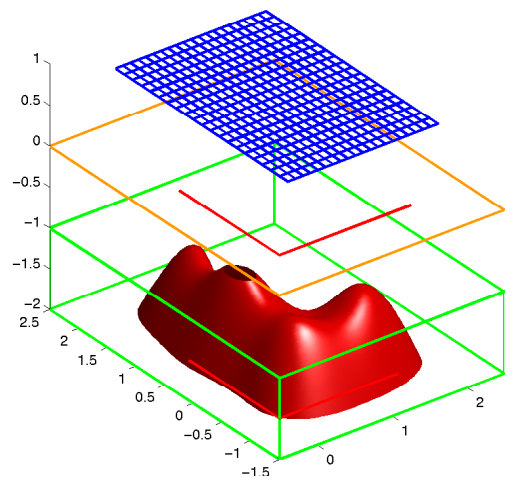

(c) Reconstructed scatterer (3D view).

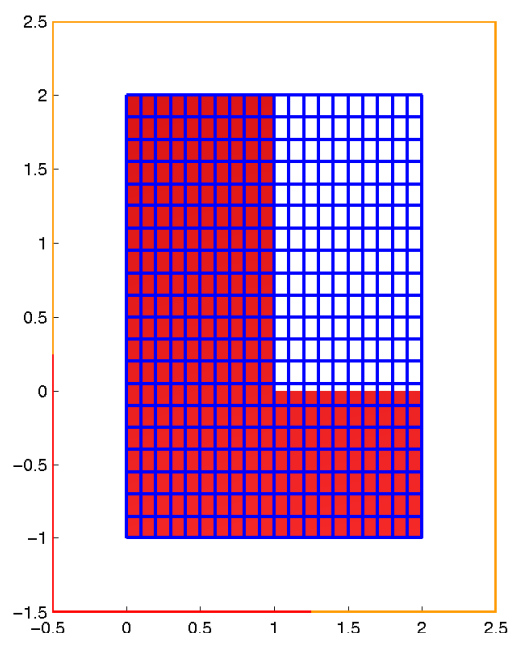

(b) Exact scatterer (view down $x_{3}$ axis).

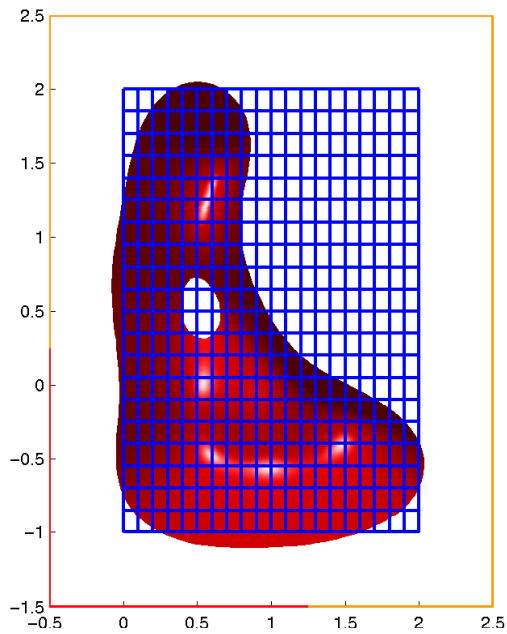

(d) Reconstructed scatterer (view down $x_{3}$ axis).

FIG. 3.4. Reconstruction of the $L$ shaped scatterer using the same measurement array and parameters as for Figure 3.2.

do not show the sphere calibration target in this case).

Although in the numerical examples here the objects are sound-soft, the same equation for $g_{z}$ (equation (3.6)) is also valid for anisotropic objects, and the support can be found in either case without knowing a priori whether or not the buried object is sound soft or anisotropic or any of the physical properties of the material (see [25] for proofs and numerical experiments in $\mathbb{R}^{2}$ ).

4. The Inverse Obstacle Problem. In this part of our paper we will be concerned with the mathematical theory of the inverse scattering problem for a sound soft obstacle. In particular, consider the direct scattering problem of finding 


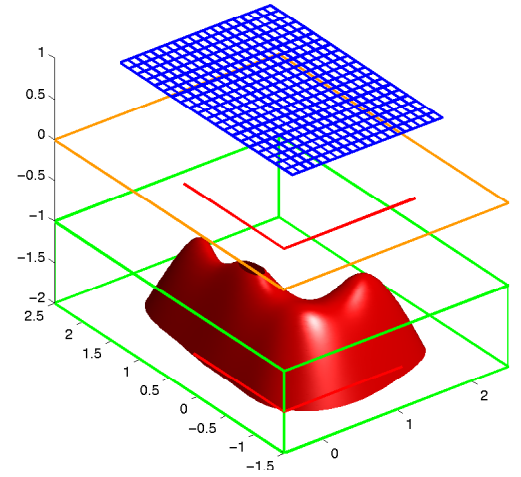

(a) Reconstruction (3D view).

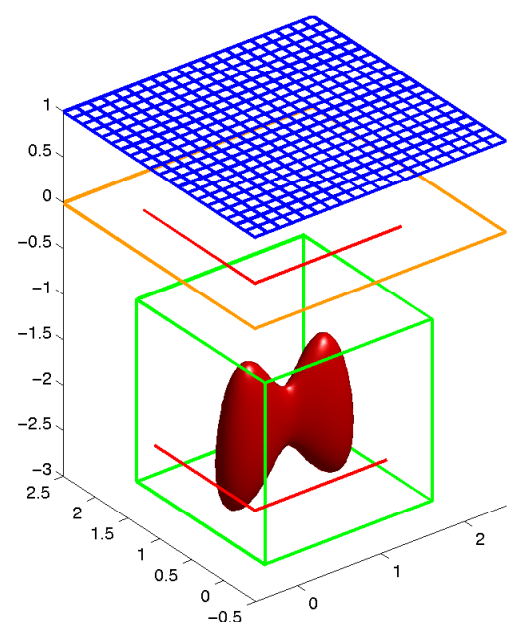

(c) Reconstruction (3D view).

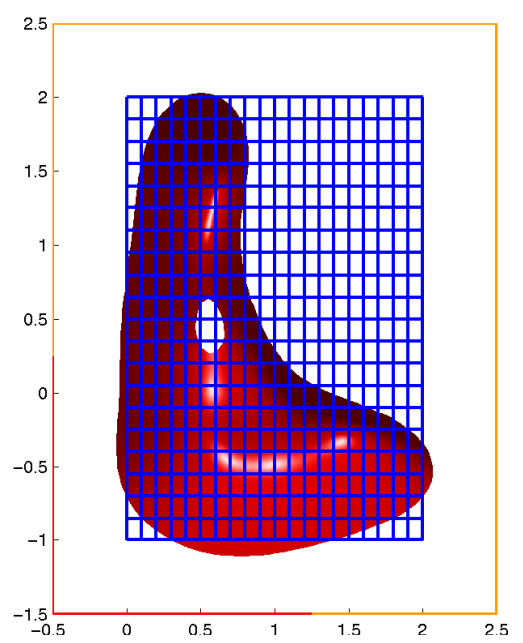

(b) Reconstruction (view down $x_{3}$ axis).

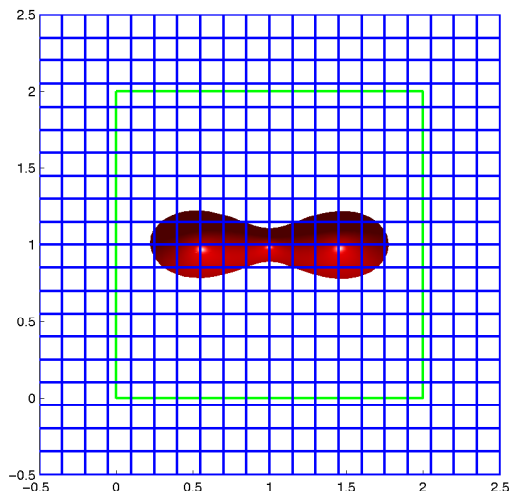

(d) Reconstruction (view down $x_{3}$ axis).

FIG. 3.5. Reconstruction of the $L$ shaped scatterer (top row) and two spheres (bottom row) when $\epsilon=0.14$ using the isovalue of 0.4. For views of the exact scatterer see Figure 3.4 and 3.3 .

$u \in C^{2}\left(\mathbb{R}^{2} \backslash \bar{D}\right) \cap C\left(\mathbb{R}^{3} \backslash D\right)$ such that

$$
\begin{gathered}
\Delta u+k^{2} u=0 \quad \text { in } \mathbb{R}^{3} \backslash \bar{D} \\
u=u^{i}+u^{s} \text { in } \mathbb{R}^{3} \backslash \bar{D} \\
u=0 \quad \text { on } \partial D \\
\lim _{r \rightarrow \infty} r\left(\frac{\partial u^{s}}{\partial r}-i k u^{s}\right)=0
\end{gathered}
$$

where $D$ is a bounded domain with connected complement $\mathbb{R}^{3} \backslash \bar{D}$ and $C^{2}$ boundary $\partial D$ having unit outward normal $\nu$, the Sommerfeld radiation condition (4.1d) is assumed 
to hold uniformly in $\hat{x}=x /|x|$ and $u^{i}(x)=e^{i k x \cdot d}$ where $d$ is a vector on the unit sphere $\Omega$. The existence of a unique solution to (4.1a)-(4.1d) is well known [10], [13]. From the Introduction we know that $u^{s}$ has the asymptotic behavior

$$
u^{s}(x)=\frac{e^{i k r}}{r} u_{\infty}(\hat{x}, d)+O\left(\frac{1}{r^{2}}\right)
$$

as $r \rightarrow \infty$ uniformly in $\hat{x}$ where $u_{\infty}$ is the far field pattern of the scattered field $u^{s}$. The inverse scattering problem we will study is that of determining $D$ from a knowledge of $u_{\infty}(\hat{x}, d)$ for $\hat{x}, d \in \Omega$. In what follows we will state the main mathematical results connected with this problem, prove some of these results, give partial proofs for others, and in some cases leave out the proofs altogether, referring the reader to the literature for details. We will follow the same procedure in Section 5. Our aim is to expand upon the brief statements given in the Introduction and attempt to give the reader a flavor of the mathematical methods used in inverse scattering theory, while avoiding some technical details.

We begin by establishing four basic results about the far field pattern and far field operator (1.11) in the case of obstacle scattering: Rellich's lemma and reciprocity for the far field pattern and the normality and injectivity properties of the far field operator. We will always assume the existence of a solution $u \in C^{2}\left(\mathbb{R}^{3} \backslash \bar{D}\right) \cap C\left(\mathbb{R}^{3} \backslash D\right)$ to the direct scattering problem (4.1a)-(4.1d) as well as the fact that since $\partial D$ is in class $C^{2}$, we have that $u \in C^{1}\left(\mathbb{R}^{3} \backslash D\right)[10]$.

THEOREM 4.1. (Rellich's Lemma): Let $u^{s}$ be a solution of the Helmholtz equation in the exterior of $D$ satisfying the Sommerfeld radiation condition (4.1d) such that the far field pattern $u_{\infty}$ of $u^{s}$ vanishes. Then $u^{s}=0$ in $\mathbb{R}^{3} \backslash \bar{D}$.

Proof. For sufficiently large $|x|$ we have a Fourier expansion

$$
u^{s}(x)=\sum_{n=0}^{\infty} \sum_{m=-n}^{n} a_{n}^{m}(r) Y_{n}^{m}(\hat{x})
$$

with respect to the spherical harmonics $Y_{n}^{m}$, where the coefficients are given by

$$
a_{n}^{m}(r)=\int_{\Omega} u(r \hat{x}) \overline{Y_{n}^{m}(\hat{x})} d s(\hat{x}) .
$$

Since $u \in C^{2}\left(\mathbb{R}^{3} \backslash \bar{D}\right)$ and the radiation condition (2.1d) holds uniformly in $\hat{x}$, we can differentiate under the integral sign and integrate by parts to conclude that $a_{n}^{m}$ is a solution of the spherical Bessel equation

$$
\frac{d^{2} a_{n}^{m}}{d r^{2}}+\frac{2}{r} \frac{d a_{n}^{m}}{d r}+\left(k^{2}-\frac{n(n+1)}{r^{2}}\right) a_{n}^{m}=0
$$

satisfying the radiation condition, i.e.

$$
a_{n}^{m}(r)=\alpha_{n}^{m} h_{n}^{(1)}(k r)
$$

where $h_{n}^{(1)}$ is a spherical Hankel function of the first kind of order $n$ and the $\alpha_{n}^{m}$ are constants depending only on $n$ and $m$. From (4.2) we have that, since $u_{\infty}=0$,

$$
\lim _{r \rightarrow \infty} \int_{|x|=r}|u(x)|^{2} d s=\int_{\Omega}\left|u_{\infty}(\hat{x})\right|^{2} d s=0 .
$$


But by Parseval's equality

$$
\int_{|x|=r}|u(x)|^{2} d s=r^{2} \sum_{n=0}^{\infty} \sum_{m=-n}^{n}\left|a_{n}^{m}(r)\right|^{2} .
$$

Substituting the above expression for $a_{n}^{m}$ into this identity, letting $r$ tend to infinity and using the asymptotic behavior of the spherical Hankel functions now yields $\alpha_{n}^{m}=0$ for all $n$ and $m$. Hence $u=0$ outside a sufficiently large sphere. By the representation formula (1.7) we see that $u$ is an analytic function of $x$ and hence we can now conclude that $u=0$ in $\mathbb{R}^{3} \backslash D$ by analyticity.

THEOREM 4.2. (Reciprocity): The far field pattern for a sound soft obstacle with incident field $u^{i}(x)=u^{i}(x, d)=e^{i k x \cdot d}$ satisfies the reciprocity relation

$$
u_{\infty}(\hat{x}, d)=u_{\infty}(-d,-\hat{x})
$$

for $\hat{x}, d \in \Omega$.

Proof. From Green's theorem

$$
\int_{D}(u \Delta v-v \Delta u) d x=\int_{\partial D}\left(u \frac{\partial v}{\partial \nu}-v \frac{\partial u}{\partial \nu}\right) d s
$$

and the asymptotic expression (4.2), we can deduce that

$$
\int_{\partial D}\left\{u^{i}(\cdot, d) \frac{\partial}{\partial \nu} u^{i}(\cdot,-\hat{x})-u^{i}(\cdot,-\hat{x}) \frac{\partial}{\partial \nu} u^{i}(\cdot, d)\right\} d s=0
$$

and

$$
\int_{\partial D}\left\{u^{s}(\cdot, d) \frac{\partial}{\partial \nu} u^{s}(\cdot,-\hat{x})-u^{s}(\cdot,-\hat{x}) \frac{\partial}{\partial \nu} u^{s}(\cdot, d)\right\} d s=0 .
$$

In the last identity we have used the fact that from Green's theorem, the integral over $\partial D$ can be replaced by an integral over the sphere $|x|=r$ for $r$ sufficiently large. From the representation (1.7) we can deduce by letting $|x| \rightarrow \infty$ that

$$
4 \pi u_{\infty}(\hat{x}, d)=\int_{\partial D}\left\{u^{s}(\cdot, d) \frac{\partial}{\partial \nu} u^{i}(\cdot,-\hat{x})-u^{i}(\cdot,-\hat{x}) \frac{\partial}{\partial \nu} u^{s}(\cdot, d)\right\} d s
$$

since the far field pattern of $\Phi(x, y)$ is $\Phi_{\infty}(\hat{x}, y)=\frac{1}{4 \pi} e^{-i k \hat{x} \cdot y}=\frac{1}{4 \pi} u^{i}(y,-\hat{x})$. Interchanging the roles of $\hat{x}$ and $d$ now gives

$$
4 \pi u_{\infty}(-d,-\hat{x})=\int_{\partial D}\left\{u^{s}(\cdot,-\hat{x}) \frac{\partial}{\partial \nu} u^{i}(\cdot, d)-u^{i}(\cdot, d) \frac{\partial}{\partial \nu} u^{s}(\cdot,-\hat{x})\right\} d s .
$$

We now subtract the last equation from the sum of the three preceding equations to obtain

$$
\begin{aligned}
& 4 \pi\left\{u_{\infty}(\hat{x}, d)-u_{\infty}(-d,-\hat{x})\right\}= \\
& \int_{\partial D}\left\{u(\cdot, d) \frac{\partial}{\partial \nu} u(\cdot,-\hat{x})-u(\cdot,-\hat{x}) \frac{\partial}{\partial \nu} u(\cdot, d)\right\} d s
\end{aligned}
$$


and the result follows from the boundary condition $u(\cdot, d)=u(\cdot,-\hat{x})=0$ on $\partial D$.

We now want to establish the fact that the far field operator $F$ defined by (1.11) where $u_{\infty}$ is the far field pattern corresponding to a sound-soft obstacle is normal, i.e. $F^{*} F=F F^{*}$ where $F^{*}$ is the adjoint operator to $F$ in $L^{2}(\Omega)$. To this end, we need the following basic identity [13], [12].

TheOrem 4.3. Let $F: L^{2}(\Omega) \rightarrow L^{2}(\Omega)$ be the far field operator corresponding to a sound-soft obstacle. Then for every $\left.g, h \in L^{2} \Omega\right)$ we have

$$
2 \pi(F g, h)=2 \pi(g, F h)+i k(F g, F h)
$$

where $(\cdot, \cdot)$ denotes the inner product on $L^{2}(\Omega)$.

Proof. If $v^{s}$ and $w^{s}$ are radiating solutions of the Helmholtz equation with far field patterns $v_{\infty}$ and $w_{\infty}$, then from the radiation condition we have that

$$
v^{s}(x) \frac{\overline{\partial w^{s}(x)}}{\partial r}=-\frac{i k}{|x|^{2}} v_{\infty}(\hat{x}, d) \overline{w_{\infty}(\hat{x}, d)}+O\left(\frac{1}{|x|^{3}}\right)
$$

as $r=|x| \rightarrow \infty$ uniformly in all directions. Hence, by Green's theorem we obtain that

$$
\int_{\partial D}\left(v^{s} \frac{\partial \overline{w^{s}}}{\partial \nu}-\overline{w^{s}} \frac{\partial v^{s}}{\partial \nu}\right) d s=-2 i k \int_{\Omega} v_{\infty} \overline{w_{\infty}} d s
$$

From the representation (1.7) we obtain

$$
v_{\infty}(\hat{x})=\frac{1}{4 \pi} \int_{\partial D}\left\{v^{s}(y) \frac{\partial e^{-i k \hat{x} \cdot y}}{\partial \nu}-\frac{\partial v^{s}}{\partial \nu}(y) e^{-i k \hat{x} \cdot y}\right\} d s(y)
$$

for $\hat{x} \in \Omega$, and hence if $w_{h}^{i}$ is a Herglotz wave function with kernel $h$ (see (1.13)), then

$$
\begin{aligned}
\int_{\partial D}\left(v^{s}(x) \frac{\partial \overline{w_{h}^{i}}}{\partial \nu}(x)\right. & \left.-\overline{w_{h}^{i}(x)} \frac{\partial v^{s}}{\partial \nu}(x)\right) d s(x) \\
& =\int_{\Omega} \overline{h(d)} \int_{\partial D}\left(v^{s}(x) \frac{\partial e^{-i k x \cdot d}}{\partial \nu}-e^{-i k \hat{x} \cdot d} \frac{\partial v^{s}}{\partial \nu}(x)\right) d s(x) d s(d) \\
& =4 \pi \int_{\Omega} \overline{h(d)} v_{\infty}(d) d s(d) .
\end{aligned}
$$

We point out to the reader that the appearance of Herglotz wave functions in our proof is due to the fact that $F h$ is the far field pattern corresponding to the incident field $w_{h}^{i}$.

Now let $v_{g}^{i}$ and $v_{h}^{i}$ be Herglotz wave functions with kernels $g, h \in L^{2}(\Omega)$ respectively and let $v_{g}, v_{h}$ be the solutions of (4.1a)-(4.1d) with $u^{i}$ replaced by $v_{g}^{i}$ and $v_{h}^{i}$ respectively. Let $v_{g}^{s}, v_{h}^{s}$ denote the scattered fields corresponding to $v_{g}$ and $v_{h}$ respectively and let $v_{g, \infty}, v_{h, \infty}$ be the corresponding far field patterns. Then from the 
boundary condition (4.1c), (4.4) and (4.6) we have

$$
\begin{aligned}
0 & =\int_{\partial D}\left(v_{g} \frac{\partial \overline{v_{h}}}{\partial \nu}-\overline{v_{h}} \frac{\partial v_{g}}{\partial \nu}\right) d s \\
& =\int_{\partial D}\left(v_{g}^{s} \frac{\partial \bar{v}_{h}^{s}}{\partial \nu}-\bar{v}_{h}^{s} \frac{\partial v_{g}^{s}}{\partial \nu}\right) d s+\int_{\partial D}\left(v_{g}^{s} \frac{\partial \bar{v}_{h}^{i}}{\partial \nu}-\bar{v}_{h}^{i} \frac{\partial v_{g}^{s}}{\partial \nu}\right) d s \\
& +\int_{\partial D}\left(v_{g}^{i} \frac{\partial \bar{v}_{h}^{s}}{\partial \nu}-\bar{v}_{h}^{s} \frac{\partial v_{g}^{i}}{\partial \nu}\right) d s \\
& =-2 i k \int_{\Omega} v_{g, \infty} \overline{v_{h, \infty}} d s+4 \pi \int_{\Omega} v_{g, \infty} \bar{h} d s-4 \pi \int_{\Omega} g \overline{v_{h, \infty}} d s \\
& =-2 i k(F g, F h)+4 \pi(F g, h)-4 \pi(g, F h)
\end{aligned}
$$

and the proof is complete. $\square$

THEOREM 4.4. (Normality): The far field operator corresponding to a soundsoft obstacle is normal.

Proof. From Theorem 4.3 we have that

$$
\left(g, i k F^{*} F h\right)=2 \pi\left\{(g, F h)-\left(g, F^{*} h\right)\right\}
$$

for all $g, h \in L^{2}(\Omega)$, and hence

$$
i k F^{*} F=2 \pi\left(F-F^{*}\right) .
$$

By reciprocity we have that

$$
\begin{aligned}
\left(F^{*} g\right)(\hat{x}) & =\int_{\Omega} \overline{u_{\infty}(d, \hat{x})} g(d) d s(d) \\
& =\int_{\Omega} \overline{u_{\infty}(-\hat{x},-d)} g(d) d s(d),
\end{aligned}
$$

and hence if we define the reflection operator $R: L^{2}(\Omega) \rightarrow L^{2}(\Omega)$ by $(R g)(d):=g(-d)$, we have that

$$
F^{*} g=\overline{R F R \bar{g}} .
$$

From this, observing that $(R g, R h)=(g, h)=(\bar{h}, \bar{g})$ for all $g, h \in L^{2}(\Omega)$, we find that

$$
\left(F^{*} g, F^{*} h\right)=(R F R \bar{h}, R F R \bar{g})=(F R \bar{h}, F R \bar{g}),
$$

and hence, using Theorem 4.3 again,

$$
\begin{aligned}
i k\left(F^{*} g, F^{*} h\right) & =2 \pi\{(F R \bar{h}, R \bar{g})-(R \bar{h}, F R \bar{g})\} \\
& =2 \pi\left\{\left(g, F^{*} h\right)-\left(F^{*} g, h\right)\right\} .
\end{aligned}
$$

If we now proceed as in the derivation of (4.7) we find that

$$
i k F F^{*}=2 \pi\left(F-F^{*}\right)
$$


and the proof is finished.

We note that if we define the scattering operator $S$ by

$$
S=I+\frac{i k}{2 \pi} F,
$$

then from (4.7) and (4.9) we see that $S S^{*}=S^{*} S=I$, i.e. for a sound-soft obstacle the scattering operator is unitary.

THEOREM 4.5. (Injectivity): The far field operator corresponding to a soundsoft obstacle is injective with dense range if and only if there does not exist a Dirichlet eigenfunction for $D$ which is a Herglotz wave function.

Proof. From (4.8) we see that $\left(F^{*} g\right)(\hat{x})=\overline{(F h)(-\hat{x})}$ where $h(d)=\overline{g(-d)}$. Hence $F$ is injective if and only if its adjoint $F^{*}$ is injective. Observing that in $L^{2}(\Omega)$ we have $N\left(F^{*}\right)^{\perp}=\overline{F\left(L^{2}(\Omega)\right)}$ for bounded operators $F$, we must only show the injectivity of $F$. To this end, we note that $F g=0$ with $g \neq 0$ is equivalent to the existence of a nontrivial Herglotz wave function $v_{g}^{i}$ with kernel $g$ for which the far field pattern of the corresponding scattered field $v^{s}$ is $v_{\infty}=0$. By Rellich's lemma (Theorem 4.1) this implies $v^{s}=0$ in $\mathbb{R}^{3} \backslash D$ and the boundary condition $v_{g}^{i}+v^{s}=0$ on $\partial D$ now shows that $v_{g}^{i}=0$ on $\partial D$. The proof is finished.

Having established the basic properties of the far field pattern and far field operator, we now turn our attention to the uniqueness of a solution to the inverse scattering problem for a sound-soft obstacle. There are two proofs of this result, due to Schiffer [49] and Kirsch and Kress [42]. Since the proof of Kirsch and Kress readily extends to scattering problems with boundary conditions other than Dirichlet's, whereas Schiffer's does not (cf. [13], p. 109), we will only consider the approach used by Kirsch and Kress (which was in turn motivated by the ideas of Isakov [34]). We begin with a simple completeness result.

Lemma 4.6. Assume that $k^{2}$ is not a Dirichlet eigenvalue for the bounded domain $B$ and $\mathbb{R}^{3} \backslash \bar{B}$ is connected. Let $u^{i}(x, d)=e^{i k x \cdot d}$. Then the restriction of the set of plane waves $\left\{u^{i}(\cdot, d): d \in \Omega\right\}$ to $\partial B$ is complete in $L^{2}(\partial B)$.

Proof. Let $\varphi \in L^{2}(\partial B)$ satisfy

$$
\int_{\partial B} \varphi(y) e^{-i k y \cdot d} d s(y)=0
$$

for all $d \in \Omega$. Then the single layer potential

$$
u(x):=\int_{\partial B} \varphi(y) \Phi(x, y) d s(y)
$$

where $\Phi$ is defined by (1.8) has vanishing far field pattern $u_{\infty}=0$. Hence by Rellich's lemma $u=0$ in $\mathbb{R}^{3} \backslash \bar{B}$. The $L^{2}$ jump relation for single layer potentials now implies that

$$
\varphi(x)-2 \int_{\partial B} \varphi(y) \frac{\partial \Phi(x, y)}{\partial \nu(x)} d s(y)=0, x \in \partial B
$$

and from this it can be shown ([13], p. 110) that $\varphi \in C(\partial B)$ and $u$ solves the homogeneous Dirichlet problem in $B$. Thus, by our assumption on $B$, we conclude that $u=0$ in $B$ and the jump relation for the normal derivative of the single layer potential now implies that $\varphi=0$. 
TheOREm 4.7. Assume that $D_{1}$ and $D_{2}$ are two sound-soft scatterers such that for a fixed wave number the far field patterns for both scatterers coincide for all incident directions $d$. Then $D_{1}=D_{2}$.

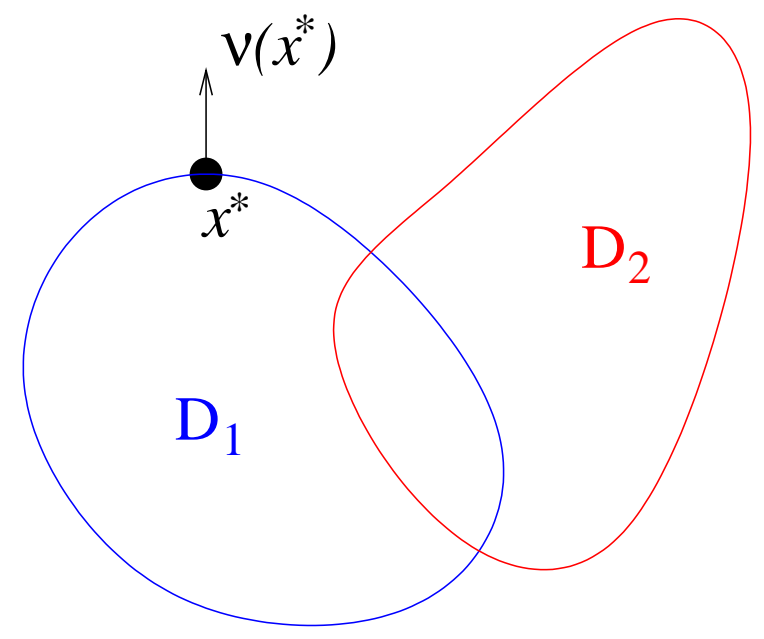

FIG. 4.1. The geometry of the scatterers considered in the proof of Theorem 4.7. We assume the scatterers are not identical and derive a contradiction.

Proof. By Rellich's lemma we can conclude that the scattered fields $u^{s}(\cdot, d)$ for the incident fields $u^{i}(x, d)=e^{i k x \cdot d}$ coincide in the unbounded component $G$ of the complement of $\bar{D}_{1} \cup \bar{D}_{2}$. Choose $x_{0} \in G$ and consider the two exterior Dirichlet problems for radiating solutions to

$$
\begin{aligned}
\Delta w_{j}^{s}+k^{2} w_{j}^{s} & =0 \text { in } \mathbb{R}^{3} \backslash \bar{D}_{j}, j=1,2 \\
w_{j}^{s}+\Phi\left(\cdot, x_{0}\right) & =0 \text { on } \partial D_{j}, j=1,2 .
\end{aligned}
$$

We will show that $w_{1}^{s}=w_{2}^{s}$ in $G$. To this end, choose a bounded domain $B$ such that $\mathbb{R}^{3} \backslash B$ is connected, $\bar{D}_{1} \cup \bar{D}_{2} \subset B, x_{0} \notin \bar{B}$ and $k^{2}$ is not a Dirichlet eigenvalue for $B$. Then by Lemma 4.6 there exists a sequence $\left(v_{n}\right)$ in $\operatorname{span}\left\{u^{i}(\cdot, d): d \in \Omega\right\}$ such that $\left\|v_{n}-\Phi\left(\cdot, x_{0}\right)\right\|_{L^{2}(\partial B)} \rightarrow 0$ as $n \rightarrow \infty$, and from potential theoretic arguments (cf. Theorem 5.4 of [13]) one can conclude that $v_{n} \rightarrow \Phi\left(\cdot, x_{0}\right)$ as $n \rightarrow \infty$, uniformly on $\bar{D}_{1} \cup \bar{D}_{2}$. Since the $v_{n}$ are linear combinations of plane waves, the corresponding scattered fields $v_{n, 1}^{s}$ and $v_{n, 2}^{s}$ for the obstacles $D_{1}$ and $D_{2}$ coincide in $G$. We can now conclude from the well-posedness of the radiating exterior Dirichlet problem that $v_{n, j}^{s} \rightarrow w_{j}^{s}, n \rightarrow \infty$, uniformly on compact subsets of $\mathbb{R}^{3} \backslash \bar{D}_{j}$ for $j=1,2$ and hence $w_{1}^{s}=w_{2}^{s}$ in $G$. Now assume that $D_{1} \neq D_{2}$. Then, without loss of generality, there exists $x^{*} \in \partial G$ such that $x^{*} \in \partial D_{1}$ and $x^{*} \notin \bar{D}_{2}$. We can choose $h>0$ such that the sequence

$$
x_{n}:=x^{*}+\frac{h}{n} \nu\left(x^{*}\right), n=1,2, \ldots
$$

is contained in $G$ and consider the solutions $w_{n, j}^{s}$ to the exterior Dirichlet problem (4.10a), (4.10b) with $x_{0}$ replaced by $x_{n}$. Then $w_{n, 1}^{s}=w_{n, 2}^{s}$ in $G$. But considering $w_{n}^{s}=w_{n, 2}^{s}$ as the scattered field corresponding to the obstacle $D_{2}$ we have that $w_{n}^{s}$ is uniformly bounded with respect to the maximum norm on closed subsets of $\mathbb{R}^{3} \backslash \bar{D}_{2}$, 
in particular $w_{n}^{s}\left(x^{*}\right)$ remains bounded as $n \rightarrow \infty$. On the other hand, considering $w_{n}^{s}=w_{n, 1}^{s}$ as the scattered field corresponding to the obstacle $D_{1}$ we have that $w_{n}^{s}\left(x^{*}\right)+\Phi\left(x^{*}, x_{n}\right)=0$ and hence $w_{n}^{s}\left(x^{*}\right)$ becomes unbounded as $n \rightarrow \infty$. This is a contradiction. Therefore $D_{1}=D_{2}$, and the proof is complete. $\square$

An open problem is to determine if one incoming plane wave for a single direction at a fixed wave number $k$ is sufficient to uniquely determine the scatterer $D$. If it is known a priori that $D$ is contained in a ball of radius $R$ and $k R<\pi$ then, as mentioned in the Introduction, it was shown by Colton and Sleeman [24] (see also Corollary, 5.3 of [13]) that a sound soft obstacle is uniquely determined by its far field pattern for a single incident direction $d$ and fixed wave number $k$.

We now turn our attention to methods for reconstructing $D$ from an inexact knowledge of the far field pattern $u_{\infty}$. We first consider the application of Newton's method. To this end we note that the solution to the direct scattering problem with a fixed incident plane wave $u^{i}$ defines an operator $\mathcal{F}: \partial D \rightarrow u_{\infty}$ which maps the boundary $\partial D$ of the sound soft scatterer $D$ onto the far field pattern $u_{\infty}$ of the scattered field. In terms of this operator, the inverse problem consists in solving the nonlinear equation $\mathcal{F}(\partial D)=u_{\infty}$. Having in mind that for ill-posed problems the norm in the data space has to be suitable for describing the measurement error, we make the assumption that $u_{\infty}$ is in the Hilbert space $L^{2}(\Omega)$. For $\partial D$ we need to choose a class of admissible surfaces described by some suitable parameterization and equipped with an appropriate norm. For the sake of simplicity, we restrict ourselves to the class of domains $D$ that are star-like with respect to the origin with $C^{2}$ boundary $\partial D$, i.e. we assume that $\partial D$ is represented in its parametric form

$$
x=r(\hat{x}) \hat{x}, \hat{x} \in \Omega
$$

for a positive function $r \in C^{2}(\Omega)$. We now view the operator $\mathcal{F}$ as a mapping from $C^{2}(\Omega)$ into $L^{2}(\Omega)$ and write $\mathcal{F}(\partial D)=u_{\infty}$ as

$$
\mathcal{F}(r)=u_{\infty} .
$$

The following basic theorem was first proved by Kirsch [37] using variational methods and subsequently by Potthast [64] using a boundary integral equation approach (see also Theorem 5.14 of [13] and [46]).

THEOREM 4.8. The operator $\mathcal{F}: r \rightarrow u_{\infty}$ is Fréchet differentiable from $C^{2}(\Omega)$ into $L^{2}(\Omega)$. The derivative is given by

$$
\mathcal{F}^{\prime} q=v_{\infty}
$$

where $v_{\infty}$ denotes the far field pattern of the solution $v^{s}$ to the Helmholtz equation in $\mathbb{R}^{3} \backslash D$ satisfying the Sommerfeld radiation condition and the boundary condition

$$
v^{s}=-\nu \cdot x_{q} \frac{\partial u}{\partial \nu} \quad \text { on } \partial D
$$

where $x_{q}=q(\hat{x}) \hat{x}$ and $\partial D$ is parameterized by $x=r(\hat{x}) \hat{x}$.

Theorem 4.8 now allows us to apply Newton's method to solve

$$
\mathcal{F}(r)=u_{\infty} .
$$

In particular, given a far field pattern $u_{\infty}$ and initial guess $r_{0}$ to $r$, the nonlinear equation $\mathcal{F}(r)=u_{\infty}$ is replaced by the linearized equation

$$
\mathcal{F}\left(r_{0}\right)+\mathcal{F}^{\prime} q=u_{\infty}
$$


which is then solved for $q$ to yield the new approximation $r_{1}$ given by $r_{1}=r_{0}+q$. Newton's method then consists in iterating this procedure [44], [47]. From Theorem 4.8 it is seen that the Fréchet derivative $\mathcal{F}^{\prime}$ is a compact operator and hence regularization methods must be used in solving (4.11), reflecting the fact that the inverse scattering problem is ill-posed. In this regard, the following theorem is important.

TheOREM 4.9. The linear operator $\mathcal{F}^{\prime}$ is injective.

Proof. Assume that $\mathcal{F}^{\prime} q=0$. Then the solution $v^{s}$ to the scattering problem stated in Theorem 4.8 has a vanishing far field pattern and hence by Rellich's lemma $v^{s}=0$ in $\mathbb{R}^{3} \backslash \bar{D}$ and consequently $v^{s}=0$ on $\partial D$. Since by Holmgren's uniqueness theorem [2] $\partial u / \partial \nu$ cannot vanish on open subsets of $\partial D$ (recall that $u=0$ on $\partial D$ ), we now have that $\nu \cdot x_{q}=0$ on $\partial D$. A short calculation now shows that this implies that $q=0$.

An alternative to Newton's method for solving the inverse scattering problem of determining a sound soft scattering obstacle from the far field pattern of the scattered field is the linear sampling method. As pointed out in the Introduction, this method has several advantages over Newton's method, although it has the disadvantage of requiring a knowledge of $u_{\infty}(\hat{x}, d)$ for all $\hat{x}, d \in \Omega$ (this assumption can be weakened see [25] and the previous section of this paper). To describe the basic idea behind the linear sampling method, assume that for every $z \in D$ there exists a unique solution $g=g(\cdot, z) \in L^{2}(\Omega)$ to the far field equation (1.12), i.e.

$$
\int_{\Omega} u_{\infty}(\hat{x}, d) g(d) d s(d)=\frac{e^{-i k \hat{x} \cdot z}}{4 \pi}
$$

where $u_{\infty}$ is the far field pattern corresponding to the scattering of the plane wave $e^{i k x \cdot d}$ by the sound soft obstacle $D$. Then, since the righthand side of (4.12) is the far field pattern of the fundamental solution $\Phi(x, z)$, it follows from Rellich's lemma that

$$
\int_{\Omega} u^{s}(x, d) g(d) d s(d)=\Phi(x, z), x \in \mathbb{R}^{3} \backslash D .
$$

From the boundary condition $u=0$ on $\partial D$ it now follows that

$$
v_{g}(x)+\Phi(x, z)=0, x \in \partial D
$$

where $v_{g}$ is the Herglotz wave function defined by (1.13). We now see from (4.13) that $v_{g}$ becomes unbounded as $z \rightarrow x \in \partial D$ and hence

$$
\lim _{z \rightarrow \partial D}\|g(\cdot, z)\|_{L^{2}(\Omega)}=\infty .
$$

Unfortunately, in general the far field equation $F g=\Phi_{\infty}(\cdot, z)$ does not have a unique solution. However, following the idea of the proof of Lemma 4.6 and using the Jacobi-Anger expansion ([13], p. 32), we can show that if $k^{2}$ is not a Dirichlet eigenvalue then for $z \in D$ the unique solution $v$ of

$$
\begin{aligned}
\Delta v+k^{2} v & =0 \text { in } D \\
v+\Phi(\cdot, z) & =0 \text { on } \partial D
\end{aligned}
$$

can be approximated in $L^{2}(\partial D)$ by a Herglotz wave function $v_{g}$. If $\partial D$ is analytic then $v$ can be uniquely continued as a solution of the Helmholtz equation to a domain 
$D^{*} \supset D$ where $k^{2}$ is not a Dirchlet eigenvalue for $D^{*}$ and this fact can be used to show that $v$ can be approximated in $C(\bar{D})$ by a Herglotz wave function [22]. We then have the following result:

TheOrem 4.10. Assume that $\partial D$ is analytic and let $z \in D$. Then for every $\epsilon>0$ there exists a solution $g=g(\cdot, z) \in L^{2}(\Omega)$ of the inequality $\left\|F g-\Phi_{\infty}(\cdot, z)\right\|_{L^{2}(\Omega)}<\epsilon$ such that

$$
\lim _{z \rightarrow \partial D}\|g(\cdot, z)\|_{L^{2}(\Omega)}=\infty
$$

and the Herglotz wave function $v_{g}$ with kernel $g$ becomes unbounded as $z \rightarrow x \in \partial D$.

The above theorem now suggests a numerical procedure for determining $\partial D$ from noisy far field data (see Section 2 of this paper). In particular, let $u_{\infty}^{\delta}$ be the measured far field data, i.e. $\left\|u_{\infty}^{\delta}-u_{\infty}\right\|<\delta$, and assume $g$ is such that $\left\|F g-\Phi_{\infty}(\cdot, z)\right\|<\epsilon$. If $F_{\delta}$ is the operator $F$ with the kernel $u_{\infty}$ replaced by $u_{\infty}^{\delta}$ then we want to find an approximation to $g$ by solving $F_{\delta} \varphi=\Phi_{\infty}(\cdot, z)$, i.e. we view both the operator and the right hand side as being inexact. For each fixed $z$ we now determine $\varphi=\varphi(\cdot, z)$ by minimizing the Tikhonov functional

$$
\left\|F_{\delta} \varphi-\Phi_{\infty}(\cdot, z)\right\|^{2}+\gamma\|\varphi(\cdot, z)\|^{2}
$$

where the regularization parameter is chosen by Morozov's generalized discrepancy principle [75], i.e. assuming that $\epsilon<<\delta, \gamma=\gamma(z)$ is chosen such that $\| F_{\delta} \varphi-$ $\Phi_{\infty}(\cdot, z)\|\approx \delta\| \varphi(\cdot, z) \|$. The unknown boundary $\partial D$ is now determined by looking for those points $z$ where $\|\varphi(\cdot, z)\|$ begins to sharply increase.

In the above theorem, the assumption that $\partial D$ is analytic is not of major concern since the far field pattern depends continuously on $C^{2}$ deformations of the boundary (cf. Theorem 4.8) and is assumed to be inexact in any case. However, a more serious problem is that nothing is said about what happens when $z \in \mathbb{R}^{3} \backslash D$. This problem was resolved by Kirsch [39], who proposed replacing the equation $F g=\Phi_{\infty}(\cdot, z)$ by $\left(F^{*} F\right)^{\frac{1}{4}} g=\Phi_{\infty}(\cdot, z)$ where $F^{*}$ is the adjoint of $F$ in $L^{2}(\Omega)$. We will now outline the main ideas of Kirsch's method. In what follows $S: L^{2}(\partial D) \rightarrow L^{2}(\partial D)$ is the single layer potential defined by

$$
(S \varphi)(x)=\int_{\partial D} \varphi(y) \Phi(x, y) d s(y), x \in \partial D
$$

and $G: L^{2}(\partial D) \rightarrow L^{2}(\Omega)$ is defined by $G h=v_{\infty}$ where $v_{\infty}$ is the far field pattern of the solution to the radiating exterior Dirichlet problem with boundary data $h \in$ $L^{2}(\partial D)$. The relation between the operators $F, G$ and $S$ is given by the following lemma [39] (see also [46]):

LEMma 4.11. The relation

$$
F=-4 \pi G S^{*} G^{*}
$$

is valid where $G^{*}: L^{2}(\Omega) \rightarrow L^{2}(\partial D)$ and $S^{*}: L^{2}(\partial D) \rightarrow L^{2}(\partial D)$ are the $L^{2}$ adjoints of $G$ and $S$, respectively.

Proof. Define the operator $H: L^{2}(\Omega) \rightarrow L^{2}(\partial D)$ by

$$
(H g)(x):=\int_{\Omega} g(d) e^{i k x \cdot d} d s(d) .
$$


Note that $H g$ is the Herglotz wave function with density $g$. The adjoint operator $H^{*}: L^{2}(\partial D) \rightarrow L^{2}(\Omega)$ is given by

$$
\left(H^{*} \varphi\right)(\hat{x})=\int_{\partial D} \varphi(y) e^{-i k \hat{x} \cdot y} d s(y)
$$

and we note that $\frac{1}{4 \pi} H^{*} \varphi$ is the far field pattern of the single layer potential (4.14). The single layer potential with continuous density $\varphi$ is continuous in $\mathbb{R}^{3}$ and thus $\frac{1}{4 \pi} H^{*} \varphi=G S \varphi$, i.e. by a denseness argument

$$
H=4 \pi S^{*} G^{*}
$$

on $L^{2}(\partial D)$. We now observe that $F g$ is the far field pattern of the solution to the radiating exterior Dirichlet problem with boundary data $-(H g)(x), x \in \partial D$, and hence

$$
F g=-G H g .
$$

Substituting (4.15) into (4.16) now yields the lemma.

We now assume that $k^{2}$ is not a Dirichlet eigenvalue for $D$. Then by Theorems 4.4 and 4.5 the far field operator $F$ is normal and one-to-one. In particular, there exist eigenvalues $\lambda_{j} \in \mathbb{C}$ of $F, j=1,2, \ldots$, with $\lambda_{j} \neq 0$ and the corresponding eigenfunctions $\psi_{j} \in L^{2}(\Omega)$ form a complete orthonormal system in $L^{2}(\Omega)$. From Theorem 4.3 we can deduce the fact that the $\lambda_{j}$ all lie on the circle of radius $2 \pi / k$ and center $2 \pi i / k$. We also note that $\left\{\left|\lambda_{j}\right|, \psi_{j}\right.$, sign $\left.\left(\lambda_{j}\right) \psi_{j}\right\}$ is a singular system of $F$ (cf. [13], p. 91) where $\operatorname{sign}\left(\lambda_{j}\right)=\lambda_{j} /\left|\lambda_{j}\right|$. By the above lemma we have that

$$
-4 \pi G S^{*} G^{*} \psi_{j}=\lambda_{j} \psi_{j} .
$$

If we define the functions $\varphi_{j} \in L^{2}(\partial D)$ by

$$
G^{*} \psi_{j}=-\sqrt{\lambda_{j}} \varphi_{j},
$$

where we choose the branch of $\sqrt{\lambda_{j}}$ such that $\operatorname{lm}\left(\sqrt{\lambda_{j}}\right)>0$ we see that

$$
G S^{*} \varphi_{j}=\frac{\sqrt{\lambda_{j}}}{4 \pi} \psi_{j}
$$

A central result of Kirsch is that the functions $\varphi_{j}$ form a Riesz basis in the Sobolev space $H^{-\frac{1}{2}}(\partial D)$, i.e. $H^{-\frac{1}{2}}(\partial D)$ consists exactly of functions $\varphi$ of the form

$$
\varphi=\sum_{j=1}^{\infty} \alpha_{j} \varphi_{j} \text { with } \sum_{j=1}^{\infty}\left|\alpha_{j}\right|^{2}<\infty
$$

We can now prove the main result of [39]:

Theorem 4.12. Assume $k^{2}$ is not a Dirichlet eigenvalue for $D$. Then the ranges of $G: H^{\frac{1}{2}}(\partial D) \rightarrow L^{2}(\Omega)$ and $\left(F^{*} F\right)^{\frac{1}{4}}$ coincide.

Proof. We use the fact that $S^{*}: H^{-\frac{1}{2}}(\partial D) \rightarrow H^{\frac{1}{2}}(\partial D)$ is an isomorphism. Suppose $G \varphi=\psi$ for some $\varphi \in H^{\frac{1}{2}}(\partial D)$. Then $\left(S^{*}\right)^{-1} \varphi \in H^{\frac{1}{2}}(\partial D)$ and thus $\left(S^{*}\right)^{-1} \varphi=\sum_{j=1}^{\infty} \alpha_{j} \varphi_{j}$ with $\sum_{j=1}^{\infty}\left|\alpha_{j}\right|^{2}<\infty$. Therefore by (2.17) we have that

$$
\psi=G \varphi=G S^{*}\left(\left(S^{*}\right)^{-1} \varphi\right)=\frac{1}{4 \pi} \sum_{j=1}^{\infty} \alpha_{j} \sqrt{\lambda_{j}} \psi_{j}=\sum_{j=1}^{\infty} \rho_{j} \psi_{j}
$$


with $\rho_{j}=\frac{1}{4 \pi} \alpha_{j} \sqrt{\lambda_{j}}$ and thus

$$
\sum_{j=1}^{\infty} \frac{\left|\rho_{j}\right|^{2}}{\left|\lambda_{j}\right|}=\frac{1}{(4 \pi)^{2}} \sum_{j=1}^{\infty}\left|\alpha_{j}\right|^{2}<\infty
$$

On the other hand, let $\psi=\sum_{j=1}^{\infty} \rho_{j} \psi_{j}$ with the $\rho_{j}$ satisfying (4.18) and define $\varphi:=$ $\sum_{j=1}^{\infty} \alpha_{j} \varphi_{j}$ with $\alpha_{j}=4 \pi \rho_{j} / \sqrt{\lambda_{j}}$. Then $\sum_{j=1}^{\infty}\left|\alpha_{j}\right|^{2}<\infty$ and hence $\varphi \in H^{-\frac{1}{2}}(\partial D), S^{*} \varphi \in$ $H^{\frac{1}{2}}(\partial D)$, and

$$
\begin{aligned}
G\left(S^{*} \varphi\right) & =\frac{1}{4 \pi} \sum_{j=1}^{\infty} \alpha_{j} \sqrt{\lambda_{j}} \psi_{j} \\
& =\sum_{j=1}^{\infty} \rho_{j} \psi_{j} \\
& =\psi
\end{aligned}
$$

Since $\sqrt{\left|\lambda_{j}\right|}$ and $\psi_{j}$ are the eigenvalues and eigenfunctions respectively of the selfadjoint operator $\left(F^{*} F\right)^{\frac{1}{4}}$, we have that

$$
R\left(\left(F^{*} F\right)^{\frac{1}{4}}\right)=\left\{\sum_{j=1}^{\infty} \rho_{j} \psi_{j}: \sum_{j=1}^{\infty} \frac{\left|\rho_{j}\right|^{2}}{\left|\lambda_{j}\right|}<\infty\right\}
$$

and as we have shown above this is precisely $R(G)$.

Since $\Phi_{\infty}(\hat{x}, z)=\frac{1}{4 \pi} e^{-i k \hat{x} \cdot z}$ is the far field pattern of the fundamental solution $\Phi(x, z)$, it is easy to verify that $\Phi_{\infty}$ is in the range of $G$ if and only if $z \in D$, i.e. $\left(F^{*} F\right)^{\frac{1}{4}} g=\Phi_{\infty}(\cdot, z)$ is solvable if and only if $z \in D$. In particular, if regularization methods are used to solve $\left(F^{*} F\right)^{\frac{1}{4}} g=\Phi_{\infty}(\cdot, z)$ then as the noise level on $u_{\infty}$ tends to zero the norm of the regularized solution remains bounded if and only if $z \in D$ [39], [75].

For further applications of spectral methods in inverse scattering theory, see Mast, et. al. [50] and Norris [56].

5. The Inverse Medium Problem. We now turn our attention to the scattering of plane waves by a penetrable inhomogeneous medium of compact support and consider the mathematical problems associated with determining either the index of refraction or the support of the inhomogeneous medium from the far field pattern of the scattered field. In particular, consider the direct scattering problem of finding $u \in C^{2}(D) \cap C^{1}(\bar{D}), u_{0} \in C^{2}\left(\mathbb{R}^{3} \backslash \bar{D}\right) \cap C^{1}\left(\mathbb{R}^{3} \backslash D\right)$ such that

$$
\begin{gathered}
\Delta u+k^{2} n(x) u=0 \quad \text { in } D \\
\Delta u_{0}+k^{2} u_{0}=0 \quad \text { in } \mathbb{R}^{3} \backslash \bar{D} \\
u_{0}(x)=e^{i k x \cdot d}+u^{s}(x) \quad \text { in } \mathbb{R}^{3} \backslash \bar{D} \\
u_{0}=u \text { on } \partial D \\
\frac{\partial u_{0}}{\partial \nu}=\alpha \frac{\partial u}{\partial \nu} \text { on } \partial D \\
\lim _{r \rightarrow \infty} r\left(\frac{\partial u^{s}}{\partial r}-i k u^{s}\right)=0
\end{gathered}
$$


where $D$ is a bounded domain with connected complement $\mathbb{R}^{3} \backslash \bar{D}$ and $C^{2}$ boundary $\partial D$ having unit outward normal $\nu, n \in C^{1}(\bar{D})$ is the index of refraction where $\operatorname{Im} n(x) \geq$ 0 for $x \in D$ such that $n(x) \neq 1$ for $x \in \partial D$ and $\alpha$ is a positive constant. As in the previous section, $d$ is a vector on the unit sphere $\Omega$ and the radiation condition (5.1f) is assumed to hold uniformly for $\hat{x}=x /|x|$ on $\Omega$. The existence of a unique solution to (5.1a)-(5.1f) has been established by Werner [78] (see also [21] and [34]). Although from a physical point of view (5.1a)-(5.1f) is somewhat restricted as far as a model for acoustic wave propagation is concerned, it suffices to demonstrate the salient features of the inverse scattering problem we want to consider.

For the direct scattering problem (5.1a)-(5.1f), there are only minor differences in the analysis for the case $\alpha=1$ and $\alpha \neq 1$. However, for the inverse scattering problem different techniques are often needed for these two cases and at the time of this writing significant questions remain for the case where $\alpha \neq 1$. Hence, for most of this section of our paper we will restrict our attention to the case where $\alpha=1$ and only mention in passing the corresponding results (or lack thereof) for the case $\alpha \neq 1$. For both cases, the basic results on the far field pattern, i.e. Rellich's lemma and the reciprocity relation, remain valid and the proofs of Theorems 4.3 and 4.4 can be easily modified to show that if $\alpha$ is real and $n$ is real valued then the far field operator is normal. However, if $\operatorname{Im} n(x)>0$ for some $x \in D$ then the far field operator $F$ is no longer normal and for both real and complex valued refractive indices the issue of injectivity of $F$ is not as simple as in the case of scattering by a sound soft obstacle. In order to discuss these issues, we now restrict our attention to the case $\alpha=1$, i.e. we will consider the scattering problem

$$
\begin{gathered}
\Delta u+k^{2} n(x) u=0 \text { in } \mathbb{R}^{3} \backslash \partial D \\
u(x)=e^{i k x \cdot d}+u^{s}(x) \\
\lim _{r \rightarrow \infty} r\left(\frac{\partial u^{s}}{\partial r}-i k u^{s}\right)=0
\end{gathered}
$$

where $u$ is twice continuously differentiable in $\mathbb{R}^{3} \backslash \partial D$ and continuously differentiable in $\mathbb{R}^{3}$.

We first turn out attention to the analogue of the basic identity in Theorem 4.3 for the case of the scattering problem (5.2a)-(5.2c) where again

$$
u^{s}(x)=\frac{e^{i k r}}{r} u_{\infty}(\hat{x}, d)+O\left(\frac{1}{r^{2}}\right)
$$

and the far field operator is defined by (1.11). Using the same notation as in Theorem 4.3 , and the fact that

$$
\int_{\partial D}\left(v_{g}^{i} \frac{\partial \overline{v_{h}^{i}}}{\partial \nu}-\overline{v_{h}^{i}} \frac{\partial v_{g}^{i}}{\partial \nu}\right) d s=0
$$


by Green's theorem, we have from Green's theorem again that

$$
\begin{aligned}
2 i k^{2} \iint_{D} \operatorname{Im} n v_{g} \overline{v_{h}} d x & =\int_{\partial D}\left(v_{g} \frac{\partial \overline{v_{h}}}{\partial \nu}-\overline{v_{h}} \frac{\partial v_{g}}{\partial \nu}\right) d s \\
& =\int_{\partial D}\left(v_{g}^{s} \frac{\partial \overline{v_{h}^{s}}}{\partial \nu}-\overline{v_{h}^{s}} \frac{\partial v_{g}^{s}}{\partial \nu}\right) d s+\int_{\partial D}\left(v_{g}^{s} \frac{\partial \overline{v_{h}^{i}}}{\partial \nu}-\overline{v_{h}^{i}} \frac{\partial v_{g}^{s}}{\partial \nu}\right) d s \\
& +\int_{\partial D}\left(v_{g}^{i} \frac{\partial \overline{v_{h}^{s}}}{\partial \nu}-\overline{v_{h}^{s}} \frac{\partial v_{g}^{i}}{\partial \nu}\right) d s .
\end{aligned}
$$

Following the proof of Theorem 4.3 now yields the following basic identity for the far field operator corresponding to the scattering problem (5.2a)-(5.2c) [13], [11]:

THEOREM 5.1. Let $v_{g}^{i}$ and $v_{h}^{i}$ be Herglotz wave functions with kernels $g, h \in L^{2}(\Omega)$ respectively and let $v_{g}, v_{h}$ be the solutions of (5.2a)-(5.2c) with $u^{i}(x)=e^{i k x \cdot d}$ replaced by $v_{g}^{i}$ and $v_{h}^{i}$ respectively. Then

$$
i k^{2} \iint_{D} \operatorname{Im} n v_{g} \overline{v_{h}} d x=2 \pi(F g, h)-2 \pi(g, F h)-i k(F g, F h)
$$

where $(\cdot, \cdot)$ denotes the inner product on $L^{2}(\Omega)$.

From Theorem 5.1, setting $g=h$ and using the fact that $v_{g}=0$ if and only if $g=0$, we see that if $\operatorname{Im} n(x)>0$ for some $x \in D$ then $F$ is injective. In particular, $F g=0$ and $\operatorname{Im} n(x)>0$ for some $x \in D$ implies by Theorem 5.1 that $v_{g}(x)=0$ for $x$ in some ball contained in $D$ and hence by unique continuation $v_{g}(x)=0$ for $x \in D$. The invertibility of the Lippmann-Schwinger equation (1.15) now implies that $v_{g}^{i}(x)=0$ for $x \in D$ and hence $g=0$. On the other hand, when $n$ is real valued we can use the following result to investigate injectivity.

THEOREM 5.2. The far field operator corresponding to (5.2a)-(5.2b) is injective with dense range if and only if there does not exist $w \in C^{2}(D) \cap C^{1}(\bar{D})$ and a Herglotz wave function $v$ such that $v, w$ is a solution to the homogeneous interior transmission problem

$$
\begin{aligned}
& \left.\begin{array}{rl}
\Delta v+k^{2} v & =0 \\
\Delta w+k^{2} n(x) w & =
\end{array}\right\} \text { in } D \\
& \left.\begin{array}{rl}
v & =w \\
\frac{\partial v}{\partial \nu} & =\frac{\partial w}{\partial \nu}
\end{array}\right\} \text { on } \partial D .
\end{aligned}
$$

Proof. As in the case of Theorem 4.5, it suffices to establish conditions for which the far field operator $F$ is injective. To this end, we note that $F g=0$ with $g \neq 0$ is equivalent to the vanishing of the far field pattern of $w^{s}$ where $w$ is the solution of (5.2a)-(5.2c) with $e^{i k x \cdot d}$ replaced by the Herglotz wave function $v$ with kernel $g$. By Rellich's lemma, $w^{s}=0$ in $\mathbb{R}^{3} \backslash D$ and hence if $w=v+w^{s}$ we have

$$
\left.\begin{array}{rl}
w & =v \\
\frac{\partial w}{\partial \nu} & =\frac{\partial v}{\partial \nu}
\end{array}\right\} \text { on } \partial D
$$

The proof is now finished. 
Knowing that the values of $k$ for which the far field operator is not injective form a discrete set is of considerable importance in the inverse scattering problem associated with (5.2a)-(5.2c) just as it is in the case of obstacle scattering, where it is known that the set of Dirichlet eigenvalues forms a discrete set. In the case of the linear sampling method, for example, this enables us to conclude that the method can fail only for a discrete set of values of $k$. From Theorem 5.2 we see that $F$ is injective if there does not exist a nontrivial solution $v, w$ to the interior transmission problem. Values of $k$ for which there exists a nontrivial solution to (5.4a), (5.4b) are called transmission eigenvalues. It was shown by Colton, Kirsch and Päiväiranta ([9] and Section 8.6 of [13]) and by Rynne and Sleeman [69] that the set of transmission eigenvalues is discrete. The analogous problem for the case when $\alpha \neq 1$ remains open.

We now turn to the problem of the unique determination of $n=n(x)$ in (5.2a)(5.2c) from a knowledge of the far field pattern $u_{\infty}(\hat{x}, d)$ for $\hat{x}, d \in \Omega$. The proof is based on the following two lemmas, where $H^{2}(B)$ denotes the usual Sobolev space on $B$ (for proofs, see [13], [32] or [38]).

LEMma 5.3. Let $B$ be an open ball centered at the origin and containing the support of $m=1-n$. Then there exists a positive constant $C$ such that for each $z \in \mathbb{C}^{3}$ with $z \cdot z=0$ and $\mid$ Re $z \mid \geq 2 k^{2}\|n\|_{\infty}$ there exists a solution $v \in H^{2}(B)$ to $\Delta v+k^{2} n v=0$ in $B$ of the form

$$
v(x)=e^{i z \cdot x}[1+r(x)]
$$

where

$$
\|r\|_{L^{2}(B)} \leq \frac{C}{|\operatorname{Re} z|}
$$

Lemma 5.4. Let $B_{1}$ and $B_{2}$ be two open balls centered at the origin and containing the support of $m=1-n$ such that $\overline{B_{1}} \subset B_{2}$. Then the set of total fields $\{u(\cdot, d), d \in \Omega\}$ satisfying (3.2a)-(3.2c) is complete in the closure of

$$
H:=\left\{v \in C^{2}\left(\overline{B_{2}}\right): \Delta v+k^{2} n v=0 \text { in } B_{2}\right\}
$$

with respect to the $L^{2}\left(B_{1}\right)$ norm.

Now we are ready to prove the following uniqueness result for the inverse medium problem.

THEOREM 5.5. The refractive index $n$ in the scattering problem (5.2a)-(5.2c) is uniquely determined by a knowledge of the far field pattern $u_{\infty}(\hat{x}, d)$ for $\hat{x}, d \in \Omega$ and a fixed wave number $k$.

Proof. Assume that $n_{1}$ and $n_{2}$ are two refractive indices such that $u_{1, \infty}(\cdot, d)=$ $u_{2, \infty}(\cdot, d), d \in \Omega$, and let $B_{1}$ and $B_{2}$ be two open balls centered at the origin and containing the supports of $1-n_{1}$ and $1-n_{2}$ such that $\bar{B}_{1} \subset B_{2}$. Then by Rellich's lemma we have that $u_{1}(\cdot, d)=u_{2}(\cdot, d)$ in $\mathbb{R}^{3} \backslash \bar{B}_{1}$ for all $d \in \Omega$. Hence $u=u_{1}-u_{2}$ satisfies $u=\partial u / \partial \nu=0$ on $\partial B_{1}$ and the differential equation

$$
\Delta u+k^{2} n_{1} u=k^{2}\left(n_{2}-n_{1}\right) u_{2} .
$$

in $B_{1}$. From this and the differential equation for $\tilde{u}_{1}=u_{1}(\cdot, \tilde{d}), \tilde{d} \in \Omega$, we obtain

$$
k^{2} \tilde{u}_{1} u_{2}\left(n_{2}-n_{1}\right)=\tilde{u}_{1}\left(\Delta u+k^{2} n_{1} u\right)=\tilde{u}_{1} \Delta u-u \Delta \tilde{u}_{1} .
$$


From Green's theorem and the fact that the Cauchy data for $u$ vanishes on $\partial B_{1}$ we now have that

$$
\iint_{B_{1}} u_{1}(\cdot, \tilde{d}) u_{2}(\cdot, d)\left(n_{1}-n_{2}\right) d x=0
$$

for all $d, \tilde{d} \in \Omega$. It follows from Lemma 5.4 that

$$
\iint_{B_{1}} v_{1} v_{2}\left(n_{1}-n_{2}\right) d x=0
$$

for all solutions $v_{1}, v_{2} \in C^{2}\left(\bar{B}_{2}\right)$ of $\Delta v_{1}+k^{2} n_{1} v_{1}=0$ and $\Delta v_{2}+k^{2} n_{2} v_{2}=0$ in $B_{2}$.

Given $y \in \mathbb{R}^{3} \backslash\{0\}$ and $\rho>0$, we now choose vectors $a, b \in \mathbb{R}^{3}$ such that $\{y, a, b\}$ is an orthogonal basis in $\mathbb{R}^{3}$ with the properties that $|a|=1$ and $|b|^{2}=|y|^{2}+\rho^{2}$. Then for $z_{1}:=y+\rho a+i b, z_{2}:=y-\rho a-i b$ we have that

$$
\begin{aligned}
z_{j} \cdot z_{j} & =\left|\operatorname{Re} z_{j}\right|^{2}-\left|\operatorname{Im} z_{j}\right|^{2}+2 i \operatorname{Re} z_{j} \cdot \operatorname{Im} z_{j} \\
& =|y|^{2}+\rho^{2}-|b|^{2} \\
& =0
\end{aligned}
$$

and

$$
\left|\operatorname{Re} z_{j}\right|^{2}=|y|^{2}+\rho^{2} \geq \rho^{2} .
$$

In (5.5) we now substitute the solutions $v_{1}$ and $v_{2}$ from Lemma 5.3 for the refractive indices $n_{1}$ and $n_{2}$ and the vectors $z_{1}$ and $z_{2}$ respectively. Since $z_{1}+z_{2}=2 y$ this gives

$$
\iint_{B_{1}} e^{2 i y \cdot x}\left[1+r_{1}(x)\right]\left[1+r_{2}(x)\right]\left[n_{1}(x)-n_{2}(x)\right] d x=0
$$

and passing to the limit as $\rho \rightarrow \infty$ gives

$$
\int_{B_{1}} \int e^{2 i y \cdot x}\left[n_{1}(x)-n_{2}(x)\right] d x=0 .
$$

Since this equation is true for arbitrary $y \in \mathbb{R}^{3}$, by the Fourier integral theorem we have that $n_{1}(x)=n_{2}(x)$ in $B_{1}$ and the proof is finished.

Uniqueness theorems for the inverse scattering problem associated with (5.1a)(5.1d) with $\alpha \neq 1$ have been given by Isakov [36], [34]. The basic idea of the proofs in this case is a combination of the above ideas together with those of Theorem 4.7 for the case of obstacle scattering.

Having established uniqueness for the inverse scattering problem, we now turn our attention to the reconstruction of the index of refraction $n$, focusing our attention on the scattering problem (5.2a)-(5.2c). As mentioned in the Introduction, there are a variety of optimization methods for reconstructing the index of refraction in this case. We shall briefly describe one of these, the dual space method, which has the advantage over other methods of being able to increase the number of incident fields without increasing the cost of solving the inverse problem. The dual space method can also be extended to the inverse scattering problem associated with (5.1a)-(5.1d) for $\alpha \neq 1[5]$. 
We begin our description of the dual space method for solving the inverse scattering problem associated with $(3.2 \mathrm{a})-(3.2 \mathrm{c})$ by assuming that there exist functions $v_{p} \in C^{2}(D) \cap C^{1}(\bar{D})$ and $w_{p} \in C^{2}(D) \cap C^{1}(\bar{D})$ which satisfy the interior transmission problem

$$
\begin{aligned}
& \left.\begin{array}{rl}
\Delta v_{p}+k^{2} v_{p} & =0 \\
\Delta w_{p}+k^{2} n(x) w_{p} & =0
\end{array}\right\} \text { in } D \\
& \left.\begin{array}{rl}
w_{p}-v_{p} & =u_{p} \\
\frac{\partial w_{p}}{\partial \nu}-\frac{\partial v_{p}}{\partial \nu} & =\frac{\partial u_{p}}{\partial \nu}
\end{array}\right\} \text { on } \partial D
\end{aligned}
$$

where

$$
u_{p}(x):=h_{p}^{(1)}(k|x|) Y_{p}(\hat{x})
$$

and $h_{p}^{(1)}$ is a spherical Hankel function of the first kind of order $p$ and $Y_{p}$ is a spherical harmonic of order $p$. If we further assume that $v_{p}$ is a Herglotz wave function written in the form

$$
v_{p}(x)=\int_{\Omega} e^{-i k x \cdot d} g_{p}(d) d s(d)
$$

where $g_{p} \in L^{2}(\Omega)$, then from the representation (4.5) for $u_{\infty}$, Green's formula and theorem and the radiation condition we have for every $d \in \Omega$ that

$$
\begin{aligned}
& \int_{\Omega} u_{\infty}(\hat{x}, d) g_{p}(\hat{x}) d s(\hat{x})= \\
& =\frac{1}{4 \pi} \int_{\partial D}\left(u^{s} \frac{\partial v_{p}}{\partial \nu}-v_{p} \frac{\partial u^{s}}{\partial \nu}\right) d s \\
& =\frac{1}{4 \pi} \int_{\partial D}\left(u \frac{\partial v_{p}}{\partial \nu}-v_{p} \frac{\partial u}{\partial \nu}\right) d s \\
& =\frac{1}{4 \pi} \int_{\partial D}\left(u \frac{\partial w_{p}}{\partial \nu}-w_{p} \frac{\partial u}{\partial \nu}\right) d s-\frac{1}{4 \pi} \int\left(u \frac{\partial u_{p}}{\partial \nu}-u_{p} \frac{\partial u}{\partial \nu}\right) d s \\
& =-\frac{1}{4 \pi} \int_{\partial D}\left(e^{i k x \cdot d} \frac{\partial u_{p}}{\partial \nu}(x)-u_{p}(x) \frac{\partial e^{i k x \cdot d}}{\partial \nu}\right) d s(x)=\frac{i^{p-1}}{k} Y_{p}(d) .
\end{aligned}
$$

We can now conclude that the identity

$$
\int_{\Omega} u_{\infty}(\hat{x}, d) g_{p}(\hat{x}) d s(\hat{x})=\frac{i^{p-1}}{k} Y_{p}(d)
$$

is satisfied if and only if there exists a solution of the interior transmission problem (5.6), (5.7) such that $v_{p}$ is a Herglotz wave function of the form (5.8). As will be shown later, a weak solution of the interior transmission problem exists if $k$ is not a transmission eigenvalue and, if $v_{p}, w_{p}$ is such a weak solution, $v_{p}$ can be approximated by a Herglotz wave function. Letting $B$ be a ball centered at the origin and containing $D$ in its interior and using Green's formula to rewrite the interior transmission problem 
(5.6), (5.7) as the operator equation (note that if $m:=1-n$ then $m(x)=0$ for $\left.x \in \mathbb{R}^{3} \backslash \bar{D}\right)$

$$
\begin{aligned}
& w_{p}+k^{2} T w_{p}=v_{p} \text { in } B \\
& u_{p}+k^{2} T w_{p}=0 \text { on } \partial B
\end{aligned}
$$

where

$$
\left(T w_{p}\right)(x):=\iint_{B} \Phi(x, y) m(y) w_{p}(y) d y
$$

and $\Phi$ is defined by (1.8) now leads to the dual space method for determining $m$ in the case when $k$ is not a transmission eigenvalue: for $0 \leq p \leq P$, determine $g_{p}$ from (5.9), define $v_{p}$ by (5.8) and then use your favorite optimization method to determine $m$ (and $w_{p}$ ) from (5.10). The first step in this procedure motivates the name dual space method since the determination of $g_{p}$ defines a linear functional on $L^{2}(\Omega)$ having prescribed values on the set of far field patterns for a fixed incident direction $d$. For further details we refer the reader to Sections 10.3 and 10.6 of [13]. If $k$ is a transmission eigenvalue, the far field equation (5.9) must be modified, leading to the concept of modified far field operators [7], [18] and the shifting of eigenvalues [11].

As we have previously mentioned, a reconstruction of the complete index of refraction is often more than is necessary. Instead, it is frequently sufficient to determine the support of $m=1-n$. This can be done by extending the linear sampling method for obstacle scattering to the case of scattering by an inhomogeneous medium. We now proceed to describe this extension for the case of problem (5.2a)-(5.2c), when $\alpha=1$. The situation when $\alpha \neq 1$ has been studied by Colton and Piana [21] and we refer the reader to this paper for details of the linear sampling method in this case (when $\alpha \neq 1$ the associated interior transmission problem is changed in an obvious way and this requires a different analysis than that which follows). As with obstacle scattering, there are two versions of the linear sampling method corresponding to the far field operator $F$ and the operator $\left(F^{*} F\right)^{\frac{1}{4}}$ respectively [8], [22], [40]. Since for arbitrary but fixed values of the wave number $k$ the method associated with $\left(F^{*} F\right)^{\frac{1}{4}}$ is restricted to non-absorbing media, i.e. $\operatorname{Im} n=0$, we only consider the far field equation $F g=\Phi_{\infty}(\cdot, z)$ which does not have this restriction. However, to avoid the problem of transmission eigenvalues we will limit our attention to the case when there exists a positive constant $c$ such that

$$
\operatorname{Im} n(x) \geq c
$$

for $x \in D$ where $\bar{D}$ is the support of $m=1-n$. If instead of (5.11) we have that Im $n(x)=0$ for $x \in D$, then the analysis which follows remains valid if we assume that $k$ is not a transmission eigenvalue.

The derivation of the linear sampling method for the inverse scattering problem associated with (5.2a)-(5.2c) is based on a projection theorem for Hilbert spaces where the inner product is replaced by a bounded sesquilinear form together with an analysis of a special interior transmission problem ([22], Section 10.7 of [13]). We begin with the projection theorem. Let $X$ be a Hilbert space with the scalar product $(\cdot, \cdot)$ and norm $\|\cdot\|$ induced by $(\cdot, \cdot)$ and let $\langle\cdot, \cdot\rangle$ be a bounded sesquilinear form on $X$ such that

$$
|\langle\varphi, \varphi\rangle| \geq C\|\varphi\|^{2}
$$


for all $\varphi \in X$ where $C$ is a positive constant. Then, using the Lax-Milgram theorem, we have the following theorem where $\oplus_{s}$ is the orthogonal decomposition with respect to the sesquilinear form $\langle\cdot, \cdot\rangle$ and $H^{\perp_{s}}$ is the orthogonal complement of $\bar{H}$ with respect to $\langle\cdot, \cdot\rangle$.

THEOREM 5.6. For every closed subspace $\bar{H} \subset X$ we have the orthogonal decomposition

$$
X=H^{\perp_{s}} \oplus_{s} \bar{H} .
$$

The projection operator $P: X \rightarrow H^{\perp_{s}}$ defined by this decomposition is bounded in $X$.

We next turn our attention to the problem of showing the existence of a unique weak solution $v, w$ of the interior transmission problem

$$
\begin{aligned}
& \left.\begin{array}{rl}
\Delta v+k^{2} v & =0 \\
\Delta w+k^{2} n(x) w & =0
\end{array}\right\} \text { in } D \\
& \left.\begin{array}{rl}
w-v & =\Phi(\cdot, z) \\
\frac{\partial w}{\partial \nu}-\frac{\partial v}{\partial \nu} & =\frac{\partial}{\partial \nu} \Phi(\cdot, z)
\end{array}\right\} \text { on } \partial D
\end{aligned}
$$

where $z \in D, n$ is assumed to satisfy (5.11), $\bar{D}$ is the support of $m=1-n$ where it is assumed that $\partial D$ is twice continuously differentiable with unit outward normal $\nu$ and $\Phi$ as usual is defined by (1.8). To motivate the following definition of a weak solution of (5.13a), (5.13b) we note that if a solution $v, w \in C^{2}(D) \cap C^{1}(\bar{D})$ to (5.13a), (5.13b) exists, then from Green's formula and Rellich's lemma we have that

$$
\begin{aligned}
w(x)+k^{2} \int_{D} \int \Phi(x, y) m(y) w(y) d y & =v(x), x \in D \\
- & k^{2} \int_{D} \int \Phi(x, y) m(y) w(y) d y=\Phi(x, z), x \in \partial B
\end{aligned}
$$

where $B$ is a ball centered at the origin with $\bar{D} \subset B$.

DeFinition 5.7. Let $H$ be the linear space of all Herglotz wave functions and $\bar{H}$ the closure of $H$ in $L^{2}(D)$. For $\varphi \in L^{2}(D)$ define the volume potential by

$$
(T \varphi)(x):=\iint_{D} \Phi(x, y) m(y) \varphi(y) d y, x \in \mathbb{R}^{3} .
$$

Then a pair $v, w$ with $v \in \bar{H}$ and $w \in L^{2}(D)$ is said to be a weak solution of the interior transmission problem (5.13a), (5.13b) with point source $z \in D$ if $v$ and $w$ satisfy the integral equation

$$
w+k^{2} T w=v
$$

and the boundary condition

$$
-k^{2} T w=\Phi(\cdot, z) \quad \text { on } \quad \partial B .
$$

The uniqueness of a weak solution to the interior transmission problem follows from a limiting argument using (3.11) and a simple application of Green's theorem 
([13], [22]). To prove existence we will use Theorem 5.6 applied to the sesquilinear form in $L^{2}(D)$ defined by

$$
\langle\varphi, \psi\rangle:=\iint_{D} m(y) \varphi(y) \overline{\psi(y)} d y
$$

and $H$ as defined in the above definition.

THEOREM 5.8. For every source point $z \in D$ there exists a weak solution to the interior transmission problem.

Proof. By a translation we can assume without loss of generality that $z=0$. We consider the space

$$
H_{1}^{0}:=\operatorname{span}\left\{j_{p}(k|x|) Y_{p}^{q}(\hat{x}), p=1,2, \cdots,-p<q \leq p\right\}
$$

and the closure $H_{1}$ of $H_{1}^{0}$ in $L^{2}(D)$ where $j_{p}$ is a spherical Bessel function and $Y_{p}^{q}$ a spherical harmonic. It can be shown that there exists a nontrivial $\psi \in H_{1}^{\perp_{s}} \cap \bar{H}$ such that $\left\langle j_{0}, \psi\right\rangle \neq 0$.

Now let $P$ be the projection operator from $L^{2}(D)$ onto $H^{\perp_{s}}$ as defined by Theorem 5.6. We first consider the integral equation

$$
u+k^{2} P T u=k^{2} P T \psi
$$

in $L^{2}(D)$. Since $T$ is compact and $P$ is bounded, the operator $P T$ is compact in $L^{2}(D)$. In order to apply the Riesz theory for compact operators [45], we will prove uniqueness for the homogeneous equation. To this end, assume that $w \in L^{2}(D)$ satisfies

$$
w+k^{2} P T w=0 .
$$

Then $w \in H^{\perp_{s}}$ and $v:=k^{2}(I-P) T w \in \bar{H}$ satisfy

$$
w+k^{2} T w=v .
$$

Since $\langle w, \varphi\rangle=0$ for all $\varphi \in H$, from the addition formula for Bessel functions we conclude that

$$
T w=0 \quad \text { on } \partial B .
$$

Hence by uniqueness of the weak interior transmission problem we have that $v=w=$ 0 . By the Riesz theory we now obtain the continuous invertibility of $I+k^{2} P T$ in $L^{2}(D)$.

Now let $u$ be the solution of (5.15) and note that $u \in H^{\perp_{s}}$. We define the constant $c$ and function $w \in L^{2}(D)$ by

$$
c:=-\frac{1}{k^{2}\left\langle j_{0}, \psi\right\rangle}, \quad w:=c(u-\psi) .
$$

Then we compute

$$
w+k^{2} P T w=-c \psi
$$

and hence

$$
w+k^{2} T w=v
$$


where $v:=k^{2}(I-P) T w-c \psi \in \bar{H}$. Since

$$
\langle h, w\rangle=c\langle h, u-\psi\rangle=0
$$

for all $h \in H_{1}$ and

$$
\left\langle j_{0}, w\right\rangle=c\left\langle j_{0}, u-\psi\right\rangle=-\frac{1}{k^{2}}
$$

we have from the addition formula for Bessel functions that

$$
-k^{2}(T w)(x)=i k h_{0}^{(1)}(k|x|)=\Phi(x, 0), x \in \partial B,
$$

where $h_{0}^{(1)}$ is a spherical Hankel function of the first kind of order zero, and the proof is complete. $\square$

We are now in a position to indicate how the support $D$ of $m$ can be determined from the far field pattern $u_{\infty}$ corresponding to the scattering problem (5.2a)-(5.2c). It suffices to determine $\partial D$. Following [8] and [22], we do this by looking for special approximate solutions of the far field equation $F g=\Phi_{\infty}(\cdot, z)$, i.e.

$$
\int_{\Omega} u_{\infty}(\hat{x}, d) g(d) d s(d)=\Phi_{\infty}(\hat{x}, z)
$$

where, as in the previous section, $\Phi_{\infty}(\cdot, z)$ is the far field pattern of the fundamental solution $\Phi(\cdot, z)$. Following the proof of Theorem 5.2 we see that $(5.16)$ has a solution if and only if the interior transmission problem (5.13a), (5.13b) with source point $z \in D$ has a solution $v, w \in C^{2}(D) \cap C^{1}(\bar{D})$ such that $v$ is a Herglotz wave function with kernel $g$. This is true only in very special cases. However, by Theorem 5.8 we know that there exists a (unique) weak solution of the interior transmission problem $v, w$ and that $v$ can be approximated in $L^{2}(D)$ by a Herglotz wave function. This fact then enables us to establish the following result analogous to Theorem 4.10 for the case of obstacle scattering [8], [22]:

TheOREM 5.9. For every $\epsilon>0$ and $z \in D$ there exists a solution $g=g(\cdot, z) \in$ $L^{2}(\Omega)$ of the inequality $\left\|F g-\Phi_{\infty}(\cdot, z)\right\|_{L^{2}(\Omega)}<\epsilon$ such that

$$
\lim _{z \rightarrow \partial D}\|g(\cdot, z)\|_{L^{2}(\Omega)}=\infty
$$

and if $v_{g}(\cdot, z)$ is the Herglotz wave function with kernel $g$ then

$$
\lim _{z \rightarrow \partial D}\left\|v_{g}(\cdot, z)\right\|_{L^{2}(D)}=\infty .
$$

As in the discussion following Theorem 4.10, the above theorem suggests a numerical procedure for determining $\partial D$ from noisy far field data (see Section 2). We note in passing that the linear sampling method has a connection with the idea of "focusing". In particular, in the time harmonic case, focusing of energy into the scatterer is accomplished by choosing the incident field to be a Herglotz wave function with kernel $g$ equal to the eigenfunction of the largest eigenvalue of the far field operator whereas in the linear sampling method the kernel $g=g(\cdot, z)$ is chosen to be (an approximate) solution of the far field equation leading to a focusing of energy on the boundary $\partial D$ of the scatterer as $z \rightarrow \partial D[50]$. 
In many, if not most, areas of application the unknown anomaly having support $D$ is situated in a piecewise homogeneous background medium rather than a homogeneous background. Furthermore, the directions of the incident fields and observation directions of the scattered field are restricted to a limited aperture as in Section 3 of this paper. For now will only address the issue of a piecewise homogeneous background medium. Following [19] and [15], we consider the scattering problem (5.2a)-(5.2c) where $k$ is piecewise constant in $\mathbb{R}^{3}$, i.e.

$$
\begin{gathered}
\Delta u+k^{2} n(x) u=0 \quad \text { in } D_{0} \\
\Delta u+k_{0}^{2} u=0 \quad \text { in } \mathbb{R}^{3} \backslash \bar{D}_{0} \\
u(x)=e^{i k_{0} x \cdot d}+u^{s}(x) \\
\lim _{r \rightarrow \infty} r\left(\frac{\partial u^{s}}{\partial r}-i k_{0} u^{s}\right)=0
\end{gathered}
$$

where $\bar{D} \subset D_{0}, n(x)=1$ for $x \in D_{0} \backslash D, D_{0}$ is bounded with a $C^{2}$ boundary $\partial D_{0}$ and $u$ is continuously differentiable across $\partial D_{0}$. We can now rewrite $(5.17 \mathrm{c})$ as

$$
u(x)=\left(e^{i k_{0} x \cdot d}+u_{b}^{s}(x)\right)+\left(u^{s}(x)-u_{b}^{s}(x)\right)
$$

where $u_{b}^{s}$ is the scattering due to the background medium alone, i.e. the scattered field for $(5.17 \mathrm{a})-(5.17 \mathrm{~d})$ in the case when $n(x)=1$ for $x \in D_{0}$.

We now let $G_{\infty}(\cdot, z)$ be the far field pattern of the Green's function $G(\cdot, z)$ for the background medium with source point $z \in D$ and consider the modified far field equation

$$
\int_{\Omega}\left[u_{\infty}(\hat{x}, d)-u_{b, \infty}^{s}(\hat{x}, d)\right] g(d) d s(d)=G_{\infty}(\hat{x}, z) .
$$

Noting that the kernel of the integral operator is the far field pattern corresponding to the incident field $u_{b}(x, d)=e^{i k_{0} x \cdot d}+u_{b}^{s}(x)$, we see from Rellich's lemma and Holmgren's uniqueness theorem [2] that if $g$ is a solution of (5.18) then

$$
U(x):=\int_{\Omega} u(x, d) g(d) d s(d)
$$

and

$$
V(x):=\int_{\Omega} u_{b}(x, d) g(d) d s(d)
$$

satisfy the interior transmission problem

$$
\begin{aligned}
& \left.\begin{array}{rl}
\Delta V+k^{2} V & =0 \\
+k^{2} n(x) U & =0
\end{array}\right\} \text { in } D \\
& U-V=G(x, z) \\
& \left.\frac{\partial U}{\partial \nu}-\frac{\partial V}{\partial \nu}=\frac{\partial}{\partial \nu} G(x, z)\right\} \text { on } \partial D \text {. }
\end{aligned}
$$

Provided we can construct a (unique) weak solution to the interior transmission problem $(5.19 \mathrm{a}),(5.19 \mathrm{~b})$, we can now prove a result analogous to Theorem 5.9 for the 


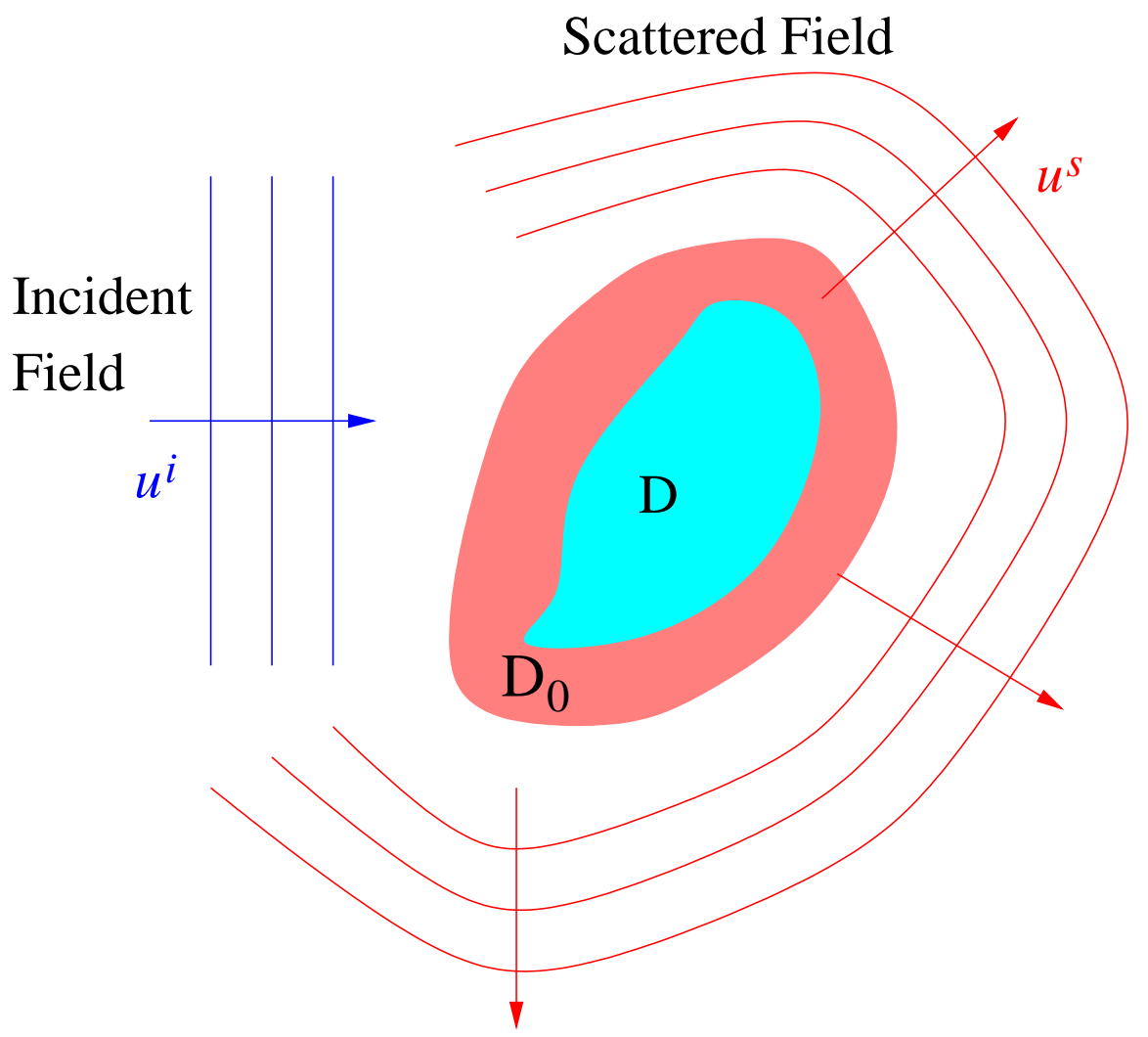

FIG. 5.1. In this section we assume that the unknown anomaly $D$ is located inside a known background medium $D_{0}$. The problem is to determine the support of $D$.

modified far field equation and thus have a method for numerically determining the support $D$ of $m=1-n$ by solving (5.18). But, for $x \in D, G(x, z)=\Phi(x, z)+h(x, z)$ where $h$ is a regular solution of the Helmholtz equation in $D$ and hence we can rewrite (5.19a), (5.19b) in the form (5.13a), (5.13b) where $w=U$ and $v=V+h$. We can now use our previous results to construct a weak solution to $(5.19 \mathrm{a}),(5.19 \mathrm{~b})$.

We conclude our survey by briefly considering the inverse scattering problem for an orthotropic medium. This problem arises if one considers the scattering of a TE polarized electromagnetic wave by an anisotropic infinite cylinder where the index of refraction has the form

$$
\left(\begin{array}{ccc}
n_{11}(x) & n_{12}(x) & 0 \\
n_{21}(x) & n_{22}(x) & 0 \\
0 & 0 & n_{33}(x)
\end{array}\right)
$$

and the $n_{i j}$ are independent of the coordinate lying along the axis of the cylinder. The magnetic field is then of the form $(0,0, u)$ where $u$ satisfies

$$
\begin{gathered}
\nabla \cdot N(x) \nabla u+k^{2} u=0 \text { in } \mathbb{R}^{2} \\
u=u^{i}+u^{s} \\
\lim _{r \rightarrow \infty} \sqrt{r}\left(\frac{\partial u^{s}}{\partial r}-i k u^{s}\right)=0
\end{gathered}
$$


with $u^{i}(x)=e^{i k x \cdot d}$ and

$$
N(x)=\frac{1}{n_{11} n_{22}-n_{12} n_{21}}\left(\begin{array}{cc}
n_{11}(x) & n_{12}(x) \\
n_{21}(x) & n_{22}(x)
\end{array}\right) .
$$

We make the assumption that $N$ is continuously differentiable in $\mathbb{R}^{2}$ such that $I-N$ has support $\bar{D} \subset \mathbb{R}^{2}$ where $D$ is a bounded domain with connected complement and $C^{2}$ boundary $\partial D$. We further assume that $M(x):=I-N(x)$ can be pointwise diagonalized with a unitary complex matrix $U(x)$ and that if $M(x)=U^{*}(x) M_{D}(x) U(x)$ where $M_{D}$ is a diagonal matrix then $I-M_{D}$ has a positive definite real part and a negative definite imaginary part. This implies that for $x \in D$ the matrix $N$ is coercive, i.e.

$$
\operatorname{Im}(a \overline{N a}) \geq \gamma(x)|a|^{2}
$$

for every $a \in \mathbb{C}^{2}$ where $\gamma(x)>0$ for $x \in D$.

Under the above assumptions, Potthast [62] was able to use the method of integral equations to show that there exists a unique solution to the direct scattering problem (5.20a)-(5.20c). From the point of view of the inverse scattering problem, the use of integral equations in solving (5.20a)-(5.20c) is crucial since this approach now enables us to follow the ideas discussed above for the isotropic case to establish the validity of the linear sampling method for determining the support of $M$. In particular, from the two dimensional version of (1.7) it follows that

$$
u^{s}(x)=\frac{e^{i k r}}{\sqrt{r}} u_{\infty}(\hat{x}, d)+O\left(\frac{1}{r^{\frac{3}{2}}}\right)
$$

as $r \rightarrow \infty$ and the inverse scattering problem of interest is to determine the support $D$ of $M$ from a knowledge of $u_{\infty}(\hat{x}, d)$ for $\hat{x}, d \in \Omega$ where $\Omega$ is now the unit circle in $\mathbb{R}^{2}$. (We note that $u_{\infty}$ does not uniquely determine $N=N(x)$ but does uniquely determine the support $D$ [14], [31], [60]). It was shown in [23] that $D$ can be determined in a manner now familiar by solving the far field equation

$$
\int_{\Omega} u_{\infty}(\hat{x}, d) g(d) d s(d)=\Phi_{\infty}(\hat{x}, z), \hat{x} \in \Omega
$$

where $\Phi_{\infty}$ is the far field pattern of the two dimensional fundamental solution

$$
\Phi(x, y):=\frac{i}{4} H_{0}^{(1)}(k|x-y|), x \neq y
$$

and $H_{0}^{(1)}$ is a Hankel function of the first kind of order zero.

The analysis in [23] establishing the validity of the linear sampling method for orthotropic media is considerably more technical than the case of isotropic media since the integral operators of concern are now strongly singular. In particular, the analysis requires that $M=I$ on $\partial D$ and this means that the proof of the existence of a unique weak solution to the interior transmission problem

$$
\begin{aligned}
& \left.\begin{array}{rl}
\Delta v+k^{2} v & =0 \\
\nabla \cdot N \nabla u+k^{2} u & =0
\end{array}\right\} \text { in } D \\
& \left.\begin{array}{rl}
u-v & =\Phi(\cdot, z) \\
\frac{\partial u}{\partial \nu}-\frac{\partial v}{\partial \nu} & =\frac{\partial}{\partial \nu} \Phi(\cdot, z)
\end{array}\right\} \text { on } \partial D
\end{aligned}
$$


must be carried out in the weighted space $L_{\Gamma}^{2}(D)$ with inner product

$$
(a, b)_{\Gamma}:=\iint_{D} a(x) \cdot \overline{\Gamma(x) b(x)} d x
$$

where $\Gamma(x)=U^{*}(x) \operatorname{Im} M_{D}(x) U(x)$. The proof of the existence of a weak solution to the interior transmission problem now requires that $\|\Gamma(x)\| \leq c$ for all $x \in D$ with a sufficiently small constant $c$. For the case when $N=N(x)$ is real, we refer the reader to $[20]$.

Acknowledgments and Disclaimer. We would like to thank the Air Force Office of Scientific Research for their support of two of us (Colton and Monk) for many years. One of us (Colton) would also like to thank the Deutsche Forschungsgemeinschaft for support at the University of Göttingen during June, 1999, where much of this manuscript was written and considerable benefit was obtained from talks with Professor Rainer Kress and Dr. Peter Hähner. We would also like to thank Professor Kress and Dr. Hähner for their careful reading of the manuscript. Finally, a particular note of thanks is due to Professors Trefethen and Papanicolaou for their help in preparing our manuscript for publication in this journal.

The effort of Colton and Monk was sponsored by the Air Force Office of Scientific Research, Air Force Materials Command, USAF, under grant number F49620-96-10039. The US Government is authorized to reproduce and distribute reprints for governmental purposes notwithstanding any copyright notation thereon. The views and conclusions contained herein are those of the authors and should not be interpreted as necessarily representing the official policies or endorsements, either expressed or implied, of the Air Force Office of Scientific Research or the US Government.

\section{REFERENCES}

[1] T. Angell, X. Jiang, and R. Kleinman, On a numerical method for inverse acoustic scattering, Inverse Problems, 13 (1997), pp. 531-545.

[2] L. Bers, F. John, And M. Schechter, Partial Differential Equations, John Wiley, New York, 1964.

[3] R. CARmichael, CRC Handbook of Physical Properties of Rocks, CRC Press, Boca Raton, Fla., 1982.

[4] Y. Chen And V. Rokhlin, On the inverse scattering problem for the Helmholtz equation in one dimension, Inverse Problems, 8 (1992), pp. 365-390.

[5] D. Colton, Far-field patterns and the inverse scattering problem for acoustic waves in an inhomogeneous medium, Quart. J. Mech. Applied Math., 42 (1989), pp. 317-326.

[6] D. Colton, K. Giebermann, And P. Monk, A regularized sampling method for solving three dimensional inverse scattering problems, SIAM J. Sci. Comp., 21 (2000), pp. 2316-2330.

[7] D. Colton And P. HäHneR, Modified far field operators in inverse scattering theory, SIAM J. Math. Anal., 24 (1993), pp. 365-389.

[8] D. Colton And A. Kirsch, A simple method for solving inverse scattering problems in the resonance region, Inverse Problems, 12 (1996), pp. 383-393.

[9] D. Colton, A. Kirsch, And L. PÄIVÄIrinta, Far field patterns for acoustic waves in an inhomogeneous medium, SIAM J. Math. Anal., 20 (1989), pp. 1472-1483.

[10] D. Colton and R. Kress, Integral Equation Methods in Scattering Theory, John Wiley, New York, 1983.

[11] — Eigenvalues of the far field operator and inverse scattering theory, SIAM J. Math. Anal., 26 (1995), pp. 601-615.

[12] - Eigenvalues of the far field operator for the Helmholtz equation in an absorbing medium, SIAM J. Appl. Math., 55 (1995), pp. 1724-1735.

[13] — Inverse Acoustic and Electromagnetic Scattering Theory, no. 93 in Applied Mathematical Sciences, Springer-Verlag, New York, second ed., 1998. 
[14] D. Colton, R. Kress, And P. Monk, Inverse scattering from an orthotropic medium, J. Comp. Applied Math., 81 (1997), pp. 269-298.

[15] D. Colton And P. Monk, A linear sampling method for the detection of leukemia using microwaves II. To appear in SIAM J. Appl. Math..

[16] — - The numerical solution of the three dimensional inverse scattering problem for timeharmonic acoustic waves, SIAM J. Sci. Stat. Comp., 8 (1987), pp. 278-291.

[17] - The inverse scattering problem for time harmonic acoustic waves in an inhomogeneous medium, Quart. Jour. Mech. Appl. Math., 41 (1988), pp. 97-125.

[18] - On a class of integral equations of the first kind in inverse scattering theory, SIAM J. Appl. Math., 53 (1993), pp. 847-860.

[19] _ - A linear sampling method for the detection of leukemia using microwaves, SIAM J. Appl. Math., 58 (1998), pp. 926-941.

[20] D. Colton And L. PÄIVÄrinta, Transmission eigenvalues and a problem of Hans Lewy. To appear in J. Comp. Applied Math.

[21] D. Colton And M. Piana, The simple method for solving the electromagnetic inverse scattering problem: The case of TE polarized waves, Inverse Problems, 14 (1998), pp. 597-614.

[22] D. Colton, M. Piana, and R. Potthast, A simple method using Morozov's discrepancy principle for solving inverse scattering problems, Inverse Problems, 13 (1997), pp. 14771493.

[23] D. Colton and R. Potthast, The inverse electromagnetic scattering problem for an anisotropic medium, Quart. Jour. Mech. Applied Math., 52 (1999), pp. 349-372.

[24] D. Colton and B. Sleeman, Uniqueness theorems for the inverse problem of acoustic scattering, IMA Jour. Appl. Math., 31 (1983), pp. 253-259.

[25] J. CoYLe, An inverse electromagnetic scattering problem in a two-layered background. To appear in Inverse Problems.

[26] J. Coyle And P. Monk, The finite element approximation of scattering in a layered medium, in Analytical and Computational Methods in Scattering and Applied Mathematic, F. Santosa and I. Stakgold, eds., no. 417 in Research Notes in Mathematics, London, 2000, Chapman \& Hall/CRC, pp. 67-84.

[27] _ Scattering of time-harmonic electromagnetic waves by anisotropic inhomogeneous scatterers or impenetrable obstacles, SIAM J. Numer. Anal., 37 (2000), pp. 1590-1617.

[28] A. Devaney, Acoustic tomography, in Inverse Problems in Acoustic and Elastic Waves, F. Santosa, et al., ed., Philadelphia, 1984, SIAM Publications, pp. 250-273.

[29] S. Gutman And M. Klibanov, Iterative method for multidimensional inverse scattering problems at fixed frequencies, Inverse Problems, 10 (1994), pp. 573-599.

[30] J. Hadamard, Lectures on Cauchy's Problem in Linear Partial Differential Equations, Yale University Press, New Haven, 1923.

[31] P. HÄHNER, On the uniqueness of the shape of a penetrable anisotropic obstacle. To appear in J. Comp. Applied Math..

[32] —_ A periodic Faddeev-type solution operator, J. Diff. Eqns., 128 (1996), pp. 300-308.

[33] F. Hetтlich, Fréchet derivatives in inverse obstacle scattering, Inverse Problems, 11 (1995), pp. 371-382.

[34] V. IsAKov, On uniqueness in the inverse transmission scattering problem, Comm. Partial Diff. Eqns., 15 (1990), pp. 1565-1587.

[35] _ Uniqueness and stability in multi-dimensional inverse problems, Inverse Problems, 9 (1993), pp. 579-621.

[36] - Inverse Problems for Partial Differential Equations, Springer-Verlag, New York, 1998.

[37] A. KIRSCH, The domain derivative and two applications in inverse scattering, Inverse Problems, 9 (1993), pp. 81-96.

[38] _ An Introduction to the Mathematical Theory of Inverse Problems, Springer-Verlag, New York, 1996.

[39] - Characterization of the shape of a scattering obstacle using the spectral data of the far field operator, Inverse Problems, 14 (1998), pp. 1489-1512.

[40] - Factorization of the far-field operator for the inhomogeneous medium case and an application in inverse scattering theory, Inverse Problems, 15 (1999), pp. 413-429.

[41] A. KIRSCH AND R. KREss, An optimization method in inverse acoustic scattering, in Boundary Elements IX, C. Brebbia, W. Wendland, and G. Kuhn, eds., Springer-Verlag, Heidelberg, 1987, pp. 3-18.

[42] - Uniqueness in inverse obstacle scattering, Inverse Problems, 9 (1993), pp. 285-299.

[43] R. Kleinman and P. van Den Berg, A modified gradient method for two dimensional problems in tomography, J. Comp. Applied Math., 42 (1992), pp. 17-35.

[44] R. KREss, Integral equation methods in inverse acoustic and electromagnetic scattering, in 
Boundary Integral Formulations for Inverse Analysis, D. Ingham and L. Wrobel, eds., Southampton, 1997, Computational Mechanics Publications, pp. 67-92.

[45] — Linear Integral Equations, Springer-Verlag, Berlin, second ed., 1999.

[46] R. Kress And L. Pärvärinta, On the far field in obstacle scattering, SIAM J. Applied Math., 59 (1999), pp. 1413-1426.

[47] R. Kress and W. Rundell, A quasi-Newton method in inverse obstacle scattering, Inverse Problems, 10 (1994), pp. 1145-1157.

[48] K. Langenberg, Applied inverse problems for acoustic, electromagnetic and elastic wave scattering, in Basic Methods of Tomography and Inverse Problems, P. Sabatier, ed., Bristol, 1987, Adam Hilger, pp. 124-467.

[49] P. Lax and R. Phillips, Scattering Theory, Academic Press, New York, 1967.

[50] T. Mast, A. NACHMAn, AND R. WANG, Focusing and imaging using eigenfunctions of the scattering operator, J. Acoust. Soc. Amer., 102 (1997), pp. 715-725.

[51] R. McGahan and R. KLeinman, Special session on image reconstruction using real data, IEEE Antennas and Propagation Magazine, 38 (1996), pp. 39-40.

[52] L. Misici AND F. Zirilli, Three dimensional inverse obstacle scattering for time harmonic acoustic waves: a numerical method, SIAM J. Sci. Stat. Comp., 15 (1994), pp. 1174-1189.

[53] S. Mitchell and S. Vavasis, Quality mesh generation in higher dimensions. To appear in SIAM J. Sci. Comp.

[54] A. Nachman, Reconstructions from boundary measurements, Annals of Math., 128 (1988), pp. $531-576$.

[55] F. NAtterer And F. WÜBbeling, A propagation-backpropagation method for ultrasound tomography, Inverse Problems, 11 (1995), pp. 1225-1232.

[56] A. NORRIS, A direct inverse scattering method for imaging obstacles with unknown surface conditions, IMA J. Applied Math., 61 (1998), pp. 267-290.

[57] R. Novikov, Multidimensional inverse spectral problems for the equation $-\Delta \psi+(v(x)-$ $E u(x)) \psi=0$, Trans. Functional Anal. Appl., 22 (1988), pp. 263-272.

[58] R. Ochs, The limited aperture problem of inverse scattering: Dirichlet boundary conditions, SIAM J. Appl. Math., 47 (1987), pp. 1320-1341.

[59] F. ODEH, Uniqueness theorems for the Helmholtz equation in domains with finite boundaries, J. Math. Mech., 12 (1963), pp. 857-867.

[60] M. PIANa, On uniqueness for anisotropic inhomogeneous inverse scattering problems, Inverse Problems, 14 (1998), pp. 1565-1579.

[61] D. Pommer, J. Morris, R. McGahan, And M. Fiddy, Imaging of unknown targets from measured scattering data, IEEE Antennas and Propagation Magazine, 41 (1999), pp. 4044.

[62] R. Pотthast, Electromagnetic scattering from an orthotropic medium. To appear in J. Int. Eqns. Appls.

[63] _ - Stability estimates and reconstructions in inverse acoustic scattering using singular sources. To appear in J. Comp. Applied Math.

[64] — Fréchet differentiability of boundary integral operators in inverse acoustic scattering, Inverse Problems, 10 (1994), pp. 431-447.

[65] — - A point-source method for inverse acoustic and electromagnetic obstacle scattering problems, IMA J. Applied. Math., 61 (1998), pp. 119-140.

[66] A. RAmm, Recovery of the potential from fixed-energy scattering data, Inverse Problems, 4 (1988), pp. 877-886.

[67] — Multidimensional Inverse Scattering Problems, Longman-Wiley, New York, 1992.

[68] A. RoGer, Newton-Kantorovich algorithm applied to an electromagnetic inverse problem, IEEE Trans. Ant. Prop., AP-29 (1981), pp. 232-238.

[69] B. Rynne And B. SleEman, The interior transmission problem and inverse scattering from inhomogeneous media, SIAM J. Math. Anal., 22 (1991), pp. 1755-1762.

[70] A. Sommerfeld, Partial Differential Equations in Physics, Academic Press, New York, 1949.

[71] J. Sylvester and G. Uhlmann, A global uniqueness theorem for an inverse boundary value problem, Annals of Math., 125 (1987), pp. 153-169.

[72] J. Sylvester And D. Winebrenner, Linear and nonlinear inverse scattering, SIAM J. Applied Math., 59 (1998), pp. 669-699.

[73] W. Tabbara, B. Duchêne, C. Pichot, D. Lesselier, L. Chommeloux, and N. JoachiMOWICZ, Diffraction tomography: Contributions to the analysis of some applications in microwaves and ultrasonics, Inverse Problems, 4 (1988), pp. 305-331.

[74] A. Tikhonov, On the solution of incorrectly formulated problems and the regularization method, Soviet Math. Doklady, 4 (1963), pp. 1035-1038.

[75] A. N. Tikhonov, A. V. Goncharsky, V. V. Stepanov, and A. G. Yagola, Numerical 
Methods for the Solution of Ill-Posed Problems, Kluwer, Dordrecht, 1995.

[76] P. VAn den Berg, B. KoolJ, and R. Kleinman, Image reconstruction from Ipswich data-III, IEEE Antennas and Propagation Magazine, 41 (1999), pp. 27-32.

[77] Y. WANG AND W. CHEW, An iterative solution of two dimensional electromagnetic inverse scattering problem, Jour. Imaging Systems and Technology, 1 (1989), pp. 100-108.

[78] P. Werner, Beugungsprobleme der mathematischen Akustik, Arch. Rat. Mech. Anal., 12 (1963), pp. 155-184.

[79] A. ZINN, On an optimization method for the full- and the limited-aperture problem in inverse acoustic scattering for a sound-soft obstacle, Inverse Problems, 5 (1989), pp. 239-253. 